\title{
SHEAF QUANTIZATION OF HAMILTONIAN \\ ISOTOPIES AND APPLICATIONS TO \\ NONDISPLACEABILITY PROBLEMS
}

STÉPHANE GUILLERMOU, MASAKI KASHIWARA, and PIERRE SCHAPIRA

\begin{abstract}
Let $I$ be an open interval containing zero, let $M$ be a real manifold, let $\dot{T}^{*} M$ be its cotangent bundle with the zero-section removed, and let $\Phi=\left\{\varphi_{t}\right\}_{t \in I}$ be a homogeneous Hamiltonian isotopy of $\dot{T}^{*} M$ with $\varphi_{0}=$ id. Let $\Lambda \subset \dot{T}^{*} M \times \dot{T}^{*} M \times T^{*} I$ be the conic Lagrangian submanifold associated with $\Phi$. We prove the existence and unicity of a sheaf $K$ on $M \times M \times I$ whose microsupport is contained in the union of $\Lambda$ and the zero-section and whose restriction to $t=0$ is the constant sheaf on the diagonal of $M \times M$. We give applications of this result to problems of nondisplaceability in contact and symplectic topology. In particular we prove that some strong Morse inequalities are stable by Hamiltonian isotopies, and we also give results of nondisplaceability for nonnegative isotopies in the contact setting.
\end{abstract}

\section{Introduction}

The microlocal theory of sheaves has been introduced and systematically developed in [11]-[13], the central idea being that of the microsupport of sheaves. More precisely, consider a real manifold $M$ of class $\mathrm{C}^{\infty}$, and consider a commutative unital ring $\mathbf{k}$ of finite global dimension. Denote by $\mathrm{D}^{\mathrm{b}}\left(\mathbf{k}_{M}\right)$ the bounded derived category of sheaves of k-modules on $M$. The microsupport $\mathrm{SS}(F)$ of an object $F$ of $\mathrm{D}^{\mathrm{b}}\left(\mathbf{k}_{M}\right)$ is a closed subset of the cotangent bundle $T^{*} M$, conic for the action of $\mathbb{R}^{+}$on $T^{*} M$ and coisotropic. Hence, this theory is "conic"; that is, it is invariant by the $\mathbb{R}^{+}$-action and is related to the homogeneous symplectic structure rather than the symplectic structure of $T^{*} M$.

To treat nonhomogeneous symplectic problems, a classical trick is to add a variable which replaces the homogeneity. This is performed for complex symplectic man-

\section{DUKE MATHEMATICAL JOURNAL}

\section{Vol. 161, No. 2, (C 2012 DOI 10.1215/00127094-1507367}

Received 30 November 2010. Revision received 22 June 2011. Accepted 1 August 2011.

2010 Mathematics Subject Classification. Primary 53D35; Secondary 14F05.

Kashiwara's work partially supported by Grant-in-Aid for Scientific Research (B) 23340005, Japan Society for the Promotion of Science. 
ifolds in [22] and later in the real case by Tamarkin [24] who adapts the microlocal theory of sheaves to the nonhomogeneous situation and deduces a new and very original proof of the classical nondisplaceability theorem conjectured by Arnold. (Tamarkin's ideas have also been exposed and developed in [9].) Note that the use of sheaf theory in symplectic topology has already appeared in [15] and [19]-[21].

In this paper, we also find a new proof of the nondisplaceability theorem and other related results, still remaining in the homogeneous symplectic framework, which makes the use of sheaf theory much easier. In other words, instead of adapting microlocal sheaf theory to treat nonhomogeneous geometrical problems, we translate these geometrical problems to homogeneous ones and apply the classical microlocal sheaf theory. Note that the converse is not always possible: there are interesting geometrical problems, for example, those related to the notion of nonnegative Hamiltonian isotopies, which make sense in the homogeneous case and which have no counterpart in the purely symplectic case.

Our main tool, as seen in the title of this paper, is a quantization of Hamiltonian isotopies in the category of sheaves. More precisely, we consider a homogeneous Hamiltonian isotopy $\Phi=\left\{\varphi_{t}\right\}_{t \in I}$ of $\dot{T}^{*} M$ (the complementary of the zero-section of $T^{*} M$ ) defined on an open interval $I$ of $\mathbb{R}$ containing zero such that $\varphi_{0}=$ id. Denoting by $\Lambda \subset \dot{T}^{*} M \times \dot{T}^{*} M \times T^{*} I$ the conic Lagrangian submanifold associated with $\Phi$, we prove that there exists a unique $K \in \mathrm{D}\left(\mathbf{k}_{M \times M \times I}\right)$ whose microsupport is contained in the union of $\Lambda$ and the zero-section of $T^{*}(M \times M \times I)$ and whose restriction to $t=0$ is the constant sheaf on the diagonal of $M \times M$.

We give a few applications of this result to problems of nondisplaceability in symplectic and contact geometry. The classical nondisplaceability conjecture of Arnold says that, on the cotangent bundle to a compact manifold $M$, the image of the zerosection of $T^{*} M$ by a Hamiltonian isotopy always intersects the zero-section. This conjecture (and its refinements, using Morse inequalities) have been proved by Chaperon [1] who treated the case of the torus by using the methods of Conley and Zehnder [6], and then by Hofer [10] and Laudenbach and Sikorav [17]. For related results in the contact case, let us quote in particular Chaperon [2], Chekanov [3], and Ferrand [8].

In this paper we recover the nondisplaceability result in the symplectic case as well as its refinement by using Morse inequalities. Indeed, we deduce these results from their homogeneous counterparts which are easy corollaries of our theorem of quantization of homogeneous Hamiltonian isotopies. We also study nonnegative Hamiltonian isotopies (which make sense only in the contact setting): we prove that given two compact connected submanifolds $X$ and $Y$ in a connected noncompact manifold $M$ and a nonnegative Hamiltonian isotopy $\Phi=\left\{\varphi_{t}\right\}_{t \in I}$ such that $\varphi_{t_{0}}$ interchanges the conormal bundle to $X$ with that of $Y$, then $X=Y$ and $\varphi_{t}$ induces the 
identity on the conormal bundle to $X$ for $t \in\left[0, t_{0}\right]$. The first part of these results has already been obtained when $X$ and $Y$ are points in [4] and [5].

\section{Microlocal theory of sheaves, after Kashiwara and Schapira [13]}

In this section, we recall some definitions and results from [13], following its notation with the exception of slight modifications. We consider a real manifold $M$ of class $\mathrm{C}^{\infty}$.

\subsection{Some geometrical notions ([13, Sections 4.2, 6.2])}

In this paper we say that a $\mathrm{C}^{1}$-map $f: M \rightarrow N$ is smooth if its differential $d_{x} f$ : $T_{x} M \rightarrow T_{f(x)} N$ is surjective for any $x \in M$. For a locally closed subset $A$ of $M$, one denotes by $\operatorname{Int}(A)$ its interior and by $\bar{A}$ its closure. One denotes by $\Delta_{M}$ or simply $\Delta$ the diagonal of $M \times M$.

One denotes by $\tau_{M}: T M \rightarrow M$ and $\pi_{M}: T^{*} M \rightarrow M$ the tangent and cotangent bundles to $M$. If $L \subset M$ is a submanifold, one denotes by $T_{L} M$ its normal bundle and $T_{L}^{*} M$ its conormal bundle. They are defined by the exact sequences

$$
\begin{gathered}
0 \rightarrow T L \rightarrow L \times_{M} T M \rightarrow T_{L} M \rightarrow 0, \\
0 \rightarrow T_{L}^{*} M \rightarrow L \times_{M} T^{*} M \rightarrow T^{*} L \rightarrow 0 .
\end{gathered}
$$

One sometimes identifies $M$ with the zero-section $T_{M}^{*} M$ of $T^{*} M$. One sets $\dot{T}^{*} M:=$ $T^{*} M \backslash T_{M}^{*} M$, and one denotes by $\dot{\pi}_{M}: \dot{T}^{*} M \rightarrow M$ the projection.

Let $f: M \rightarrow N$ be a morphism of real manifolds. To $f$ are associated the tangent morphisms

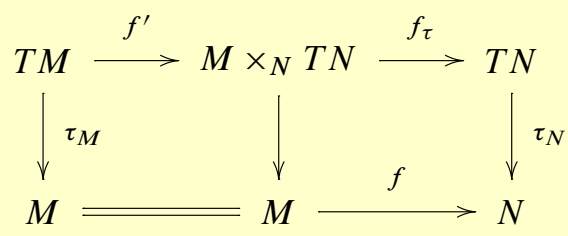

By duality, we deduce the diagram:

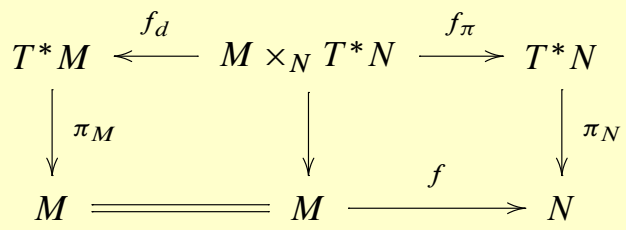

One sets

$$
T_{M}^{*} N:=\operatorname{Ker} f_{d}=f_{d}^{-1}\left(T_{M}^{*} M\right) \subset M \times_{N} T^{*} N .
$$


Note that, denoting by $\Gamma_{f}$ the graph of $f$ in $M \times N$, the projection $T^{*}(M \times N) \rightarrow$ $M \times T^{*} N$ identifies $T_{\Gamma_{f}}^{*}(M \times N)$ and $M \times{ }_{N} T^{*} N$.

Now consider the homogeneous symplectic manifold $T^{*} M$ : it is endowed with the Liouville 1-form given in a local homogeneous symplectic coordinate system $(x ; \xi)$ on $T^{*} M$ by

$$
\alpha_{M}=\langle\xi, d x\rangle .
$$

The antipodal map $a_{M}$ is defined by

$$
a_{M}: T^{*} M \rightarrow T^{*} M, \quad(x ; \xi) \mapsto(x ;-\xi) .
$$

If $A$ is a subset of $T^{*} M$, we denote by $A^{a}$ instead of $a_{M}(A)$ its image by the antipodal map.

We use the Hamiltonian isomorphism $H: T^{*}\left(T^{*} M\right) \stackrel{\sim}{\longrightarrow} T\left(T^{*} M\right)$ given in a local symplectic coordinate system $(x ; \xi)$ by

$$
H(\langle\lambda, d x\rangle+\langle\mu, d \xi\rangle)=-\left\langle\lambda, \partial_{\xi}\right\rangle+\left\langle\mu, \partial_{x}\right\rangle .
$$

\subsection{Microsupport ([13, Sections 5.1, 6.5])}

We consider a commutative unital ring $\mathbf{k}$ of finite global dimension (e.g., $\mathbf{k}=\mathbb{Z}$ ). (We assume that $\mathbf{k}$ is a field when we use Morse inequalities in Section 4.) We denote by $\mathrm{D}\left(\mathbf{k}_{M}\right)$ (resp., $\mathrm{D}^{\mathrm{b}}\left(\mathbf{k}_{M}\right)$ ) the derived category (resp., bounded derived category) of sheaves of $\mathbf{k}$-modules on $M$. We denote by $\omega_{M} \in \mathrm{D}^{\mathrm{b}}\left(\mathbf{k}_{M}\right)$ the dualizing complex on $M$. Recall that $\omega_{M}$ is isomorphic to the orientation sheaf shifted by the dimension. We recall the definition of the microsupport (or singular support) $\mathrm{SS}(F)$ of a sheaf $F$ (see [13, Definition 5.1.2]).

\section{Definition 1.1}

Let $F \in \mathrm{D}^{\mathrm{b}}\left(\mathbf{k}_{M}\right)$, and let $p \in T^{*} M$. One says that $p \notin \mathrm{SS}(F)$ if there exists an open neighborhood $U$ of $p$ such that for any $x_{0} \in M$ and any real $\mathrm{C}^{1}$-function $\varphi$ on $M$ defined in a neighborhood of $x_{0}$ with $\left(x_{0} ; d \varphi\left(x_{0}\right)\right) \in U$, one has

$$
\mathrm{R} \Gamma_{\left\{x ; \varphi(x) \geq \varphi\left(x_{0}\right)\right\}}(F)_{x_{0}} \simeq 0 .
$$

In other words, $p \notin \mathrm{SS}(F)$ if the sheaf $F$ has no cohomology supported by "halfspaces" whose conormals are contained in a neighborhood of $p$.

- $\quad$ By its construction, the microsupport is $\mathbb{R}^{+}$-conic, that is, invariant by the action of $\mathbb{R}^{+}$on $T^{*} M$.

- We have $\operatorname{SS}(F) \cap T_{M}^{*} M=\pi_{M}(\operatorname{SS}(F))=\operatorname{Supp}(F)$.

- The microsupport satisfies the triangular inequality: if $F_{1} \rightarrow F_{2} \rightarrow F_{3} \stackrel{+1}{\longrightarrow}$ is a distinguished triangle in $\mathrm{D}^{\mathrm{b}}\left(\mathbf{k}_{M}\right)$, then $\operatorname{SS}\left(F_{i}\right) \subset \mathrm{SS}\left(F_{j}\right) \cup \operatorname{SS}\left(F_{k}\right)$ for all $i, j, k \in\{1,2,3\}$ with $j \neq k$. 
In the sequel, for a locally closed subset $Z$ of $M$, we denote by $\mathbf{k}_{Z}$ the constant sheaf with stalk $\mathbf{k}$ on $Z$, extended by zero on $M \backslash Z$.

\section{Example 1.2}

(i) If $F$ is a nonzero local system on $M$ and $M$ is connected, then $\operatorname{SS}(F)=$ $T_{M}^{*} M$.

(ii) If $N$ is a closed submanifold of $M$ and $F=\mathbf{k}_{N}$, then $\operatorname{SS}(F)=T_{N}^{*} M$, the conormal bundle to $N$ in $M$.

(iii) Let $\varphi$ be a $\mathrm{C}^{1}$-function such that $d \varphi(x) \neq 0$ whenever $\varphi(x)=0$. Let $U=$ $\{x \in M ; \varphi(x)>0\}$, and let $Z=\{x \in M ; \varphi(x) \geq 0\}$. Then

$$
\begin{aligned}
& \operatorname{SS}\left(\mathbf{k}_{U}\right)=U \times_{M} T_{M}^{*} M \cup\{(x ; \lambda d \varphi(x)) ; \varphi(x)=0, \lambda \leq 0\}, \\
& \operatorname{SS}\left(\mathbf{k}_{Z}\right)=Z \times_{M} T_{M}^{*} M \cup\{(x ; \lambda d \varphi(x)) ; \varphi(x)=0, \lambda \geq 0\} .
\end{aligned}
$$

For a precise definition of being involutive (or coisotropic), we refer to [13, Definition 6.5.1].

THEOREM 1.3

Let $F \in \mathrm{D}^{\mathrm{b}}\left(\mathbf{k}_{M}\right)$. Then its microsupport $\mathrm{SS}(F)$ is involutive.

\subsection{Localization ([13, Section 6.1])}

Now let $A$ be a subset of $T^{*} M$, and let $Z=T^{*} M \backslash A$. The full subcategory $\mathrm{D}_{Z}^{\mathrm{b}}\left(\mathbf{k}_{M}\right)$ of $\mathrm{D}^{\mathrm{b}}\left(\mathbf{k}_{M}\right)$ consisting of sheaves $F$ such that $\mathrm{SS}(F) \subset Z$ is triangulated. One sets

$$
\mathrm{D}^{\mathrm{b}}\left(\mathbf{k}_{M} ; A\right):=\mathrm{D}^{\mathrm{b}}\left(\mathbf{k}_{M}\right) / \mathrm{D}_{Z}^{\mathrm{b}}\left(\mathbf{k}_{M}\right),
$$

the localization of $\mathrm{D}^{\mathrm{b}}\left(\mathbf{k}_{M}\right)$ by $\mathrm{D}_{Z}^{\mathrm{b}}\left(\mathbf{k}_{M}\right)$. Hence, the objects of $\mathrm{D}^{\mathrm{b}}\left(\mathbf{k}_{M} ; A\right)$ are those of $\mathrm{D}^{\mathrm{b}}\left(\mathbf{k}_{M}\right)$, but a morphism $u: F_{1} \rightarrow F_{2}$ in $\mathrm{D}^{\mathrm{b}}\left(\mathbf{k}_{M}\right)$ becomes an isomorphism in $\mathrm{D}^{\mathrm{b}}\left(\mathbf{k}_{M} ; A\right)$ if, after embedding this morphism in a distinguished triangle $F_{1} \rightarrow F_{2} \rightarrow$ $F_{3} \stackrel{+1}{\longrightarrow}$, one has $\operatorname{SS}\left(F_{3}\right) \cap A=\emptyset$.

When $A=\{p\}$ for some $p \in T^{*} M$, one simply writes $\mathrm{D}^{\mathrm{b}}\left(\mathbf{k}_{M} ; p\right)$ instead of $\mathrm{D}^{\mathrm{b}}\left(\mathbf{k}_{M} ;\{p\}\right)$.

\subsection{Functorial operations ([13, Section 5.4])}

Let $M$ and $N$ be two real manifolds. We denote by $q_{i}(i=1,2)$ the $i$ th projection defined on $M \times N$ and by $p_{i}(i=1,2)$ the $i$ th projection defined on $T^{*}(M \times N) \simeq$ $T^{*} M \times T^{*} N$.

\section{Definition 1.4}

Let $f: M \rightarrow N$ be a morphism of manifolds, and let $\Lambda \subset T^{*} N$ be a closed $\mathbb{R}^{+}$-conic subset. One says that $f$ is noncharacteristic for $\Lambda$ (or else, $\Lambda$ is noncharacteristic 
for $f$ ) if

$$
f_{\pi}^{-1}(\Lambda) \cap T_{M}^{*} N \subset M \times{ }_{N} T_{N}^{*} N
$$

If $\Lambda$ is a closed $\mathbb{R}^{+}$-conic subset of $\dot{T}^{*} N$, we say that $\Lambda$ is noncharacteristic for $f$ if so is $\Lambda \cup T_{N}^{*} N$.

A morphism $f: M \rightarrow N$ is noncharacteristic for a closed $\mathbb{R}^{+}$-conic subset $\Lambda$ if and only if $f_{d}: M \times_{N} T^{*} N \rightarrow T^{*} M$ is proper on $f_{\pi}^{-1}(\Lambda)$ and in this case $f_{d} f_{\pi}^{-1}(\Lambda)$ is closed and $\mathbb{R}^{+}$-conic in $T^{*} M$.

\section{THEOREM 1.5 (See [13, Section 5.4])}

Let $f: M \rightarrow N$ be a morphism of manifolds, let $F \in \mathrm{D}^{\mathrm{b}}\left(\mathbf{k}_{M}\right)$, and let $G \in \mathrm{D}^{\mathrm{b}}\left(\mathbf{k}_{N}\right)$.

(i) Assume that $f$ is proper on $\operatorname{Supp}(F)$. Then $\operatorname{SS}\left(\mathrm{R} f_{!} F\right) \subset f_{\pi} f_{d}^{-1} \operatorname{SS}(F)$.

(ii) Assume that $f$ is noncharacteristic for $\operatorname{SS}(G)$. Then $\operatorname{SS}\left(f^{-1} G\right) \subset$ $f_{d} f_{\pi}^{-1} \mathrm{SS}(G)$.

(iii) Assume that $f$ is smooth. Then $\mathrm{SS}(F) \subset M \times_{N} T^{*} N$ if and only if for any $j \in \mathbb{Z}$, the sheaves $H^{j}(F)$ are locally constant on the fibers of $f$.

There exist estimates of the microsupport for characteristic inverse images and (in some special situations) for nonproper direct images, but we do not use them here.

\section{COROLLARY 1.6}

Let I be a contractible manifold, and let $p: M \times I \rightarrow M$ be the projection. If $F \in$ $\mathrm{D}^{\mathrm{b}}\left(\mathbf{k}_{M \times I}\right)$ satisfies $\mathrm{SS}(F) \subset T^{*} M \times T_{I}^{*} I$, then $F \simeq p^{-1} \mathrm{R} p_{*} F$.

\section{Proof}

The result follows from Theorem 1.5(iii) and [13, Proposition 2.7.8].

\section{COROLLARY 1.7}

Let $I$ be an open interval of $\mathbb{R}$, and let $q: M \times I \rightarrow I$ be the projection. Let $F \in$ $\mathrm{D}^{\mathrm{b}}\left(\mathbf{k}_{M \times I}\right)$ such that $\mathrm{SS}(F) \cap T_{M}^{*} M \times T^{*} I \subset T_{M \times I}^{*}(M \times I)$ and $q$ is proper on $\operatorname{Supp}(F)$. Then, setting $F_{a}:=\left.F\right|_{\{t=a\}}$, we have isomorphisms $\mathrm{R} \Gamma\left(M ; F_{S}\right) \simeq \mathrm{R} \Gamma(M$; $F_{t}$ ) for any $s, t \in I$.

\section{Proof}

It follows from Theorem 1.5 that $\operatorname{SS}\left(\operatorname{R} q_{*} F\right) \subset T_{I}^{*} I$. Therefore, there exists $M \in$ $\mathrm{D}^{\mathrm{b}}(\mathbf{k})$ and an isomorphism $\mathrm{R} q_{*} F \simeq M_{I}$. (Recall that $M_{I}=a_{I}^{-1} M$, where $a_{I} \rightarrow$ $\{\mathrm{pt}\}$ is the projection and $M$ is identified to a sheaf on $\{\mathrm{pt}\}$.) Since $\operatorname{R\Gamma }\left(M ; F_{s}\right) \simeq$ $\left(\mathrm{R} q_{*} F\right)_{s}$, the result follows. 
1.5. Morse lemma and Morse inequalities ([13, Section 5.4])

In this subsection, we consider a function $\psi: M \rightarrow \mathbb{R}$ of class $\mathrm{C}^{1}$. We set

$$
\Lambda_{\psi}=\{(x ; d \psi(x))\} \subset T^{*} M .
$$

The proposition below is a particular case of the microlocal Morse lemma (see [13, Corollary 5.4.19]) and follows from Theorem 1.5(ii). The classical theory corresponds to the constant sheaf $F=\mathbf{k}_{M}$.

PROPOSITION 1.8

Let $F \in \mathrm{D}^{\mathrm{b}}\left(\mathbf{k}_{M}\right)$, let $\psi: M \rightarrow \mathbb{R}$ be a function of class $\mathrm{C}^{1}$, and assume that $\psi$ is proper on $\operatorname{Supp}(F)$. Let $a<b$ in $\mathbb{R}$, and assume that $d \psi(x) \notin \operatorname{SS}(F)$ for $a \leq \psi(x)<$ b. For $t \in \mathbb{R}$, set $M_{t}=\psi^{-1}(]-\infty, t[)$. Then the restriction morphism $\mathrm{R} \Gamma\left(M_{b} ; F\right) \rightarrow$ $\mathrm{R} \Gamma\left(M_{a} ; F\right)$ is an isomorphism.

\section{COROLLARY 1.9}

Let $F \in \mathrm{D}^{\mathrm{b}}\left(\mathbf{k}_{M}\right)$, and let $\psi: M \rightarrow \mathbb{R}$ be a function of class $\mathrm{C}^{1}$. Let $\Lambda_{\psi}$ be as in (1.4). Assume that

(i) $\operatorname{Supp}(F)$ is compact,

(ii) $\mathrm{R} \Gamma(M ; F) \neq 0$.

Then $\Lambda_{\psi} \cap \mathrm{SS}(F) \neq \emptyset$.

Until the end of this subsection as well as in Section 4.4 we assume that $\mathbf{k}$ is a field. The classical Morse inequalities are extended to sheaves (see [23], [13, Proposition 5.4.20]). Let us briefly recall this result.

For a bounded complex $E$ of $\mathbf{k}$-vector spaces with finite-dimensional cohomologies, we set

$$
b_{j}(E)=\operatorname{dim} H^{j}(E), \quad b_{l}^{*}(E)=(-1)^{l} \sum_{j \leq l}(-1)^{j} b_{j}(E) .
$$

We consider a map $\psi: M \rightarrow \mathbb{R}$ of class $\mathrm{C}^{1}$ and define $\Lambda_{\psi}$ as above. Note that we do not ask $\psi$ to be smooth. Let $F \in \mathrm{D}^{\mathrm{b}}\left(\mathbf{k}_{M}\right)$. Assume that

$$
\text { the set } \Lambda_{\psi} \cap \operatorname{SS}(F) \text { is finite, say, }\left\{p_{1}, \ldots, p_{N}\right\}
$$

and, setting

$$
x_{i}=\pi\left(p_{i}\right), \quad V_{i}:=\left(\mathrm{R} \Gamma_{\left\{\psi(x) \geq \psi\left(x_{i}\right)\right\}}(F)\right)_{x_{i}},
$$

also assume that

$$
\text { for all } i \in\{1, \ldots, N\}, j \in \mathbb{Z} \text {, the spaces } H^{j}\left(V_{i}\right) \text { are finite-dimensional. }
$$


Set

$$
b_{j}(F)=b_{j}(\mathrm{R} \Gamma(M ; F)), \quad b_{j}^{*}(F)=b_{j}^{*}(\mathrm{R} \Gamma(M ; F)) .
$$

THEOREM 1.10

Let $F \in \mathrm{D}^{\mathrm{b}}\left(\mathbf{k}_{M}\right)$, and assume that $\operatorname{Supp}(F)$ is compact. Assume moreover (1.5) and (1.7). Then

$$
b_{l}^{*}(F) \leq \sum_{i=1}^{N} b_{l}^{*}\left(V_{i}\right) \quad \text { for any } l
$$

Notice that (1.8) immediately implies

$$
b_{j}(F) \leq \sum_{i=1}^{N} b_{j}\left(V_{i}\right) \text { for any } j .
$$

\subsection{Kernels ([13, Section 3.6])}

Let $M_{i}(i=1,2,3)$ be manifolds. For short, we write $M_{i j}:=M_{i} \times M_{j}(1 \leq i$, $j \leq 3$ ) and $M_{123}=M_{1} \times M_{2} \times M_{3}$. We denote by $q_{i}$ the projection $M_{i j} \rightarrow M_{i}$ or the projection $M_{123} \rightarrow M_{i}$ and by $q_{i j}$ the projection $M_{123} \rightarrow M_{i j}$. Similarly, we denote by $p_{i}$ the projection $T^{*} M_{i j} \rightarrow T^{*} M_{i}$ or the projection $T^{*} M_{123} \rightarrow T^{*} M_{i}$ and by $p_{i j}$ the projection $T^{*} M_{123} \rightarrow T^{*} M_{i j}$. We also need to introduce the map $p_{12^{a}}$, the composition of $p_{12}$ and the antipodal map on $T^{*} M_{2}$.

Let $\Lambda_{1} \subset T^{*} M_{12}$ and $\Lambda_{2} \subset T^{*} M_{23}$. We set

$$
\Lambda_{1} \circ \Lambda_{2}:=p_{13}\left(p_{12^{a}}-1 \Lambda_{1} \cap p_{23}^{-1} \Lambda_{2}\right)
$$

We consider the operation of convolution of kernels:

$$
\begin{aligned}
\underset{M_{2}}{\circ}: \mathrm{D}^{\mathrm{b}}\left(\mathbf{k}_{M_{12}}\right) \times \mathrm{D}^{\mathrm{b}}\left(\mathbf{k}_{M_{23}}\right) & \rightarrow \mathrm{D}^{\mathrm{b}}\left(\mathbf{k}_{M_{13}}\right) \\
\left(K_{1}, K_{2}\right) & \mapsto K_{1} \underset{M_{2}}{\circ} K_{2}:=\mathrm{R} q_{13 !}\left(q_{12}^{-1} K_{1} \otimes q_{23}^{-1} K_{2}\right) .
\end{aligned}
$$

Let $\Lambda_{i}=\mathrm{SS}\left(K_{i}\right) \subset T^{*} M_{i, i+1}$, and assume that

$$
\left\{\begin{array}{c}
\text { (i) } q_{13} \text { is proper on } q_{12}^{-1} \operatorname{Supp}\left(K_{1}\right) \cap q_{23}^{-1} \operatorname{Supp}\left(K_{2}\right), \\
\text { (ii) } p_{12^{a}}^{-1} \Lambda_{1} \cap p_{23}^{-1} \Lambda_{2} \cap\left(T_{M_{1}}^{*} M_{1} \times T^{*} M_{2} \times T_{M_{3}}^{*} M_{3}\right) \\
\subset T_{M_{1} \times M_{2} \times M_{3}}^{*}\left(M_{1} \times M_{2} \times M_{3}\right)
\end{array}\right.
$$

It follows from Theorem 1.5 that under the assumption (1.11) we have 


$$
\operatorname{SS}\left(K_{1} \underset{M_{2}}{\circ} K_{2}\right) \subset \Lambda_{1} \circ \Lambda_{2} .
$$

If there is no risk of confusion, we write $\circ$ instead of $\stackrel{\circ}{\mathrm{M}_{2}}$.

We also use a relative version of the convolution of kernels. For a manifold $I$, $K_{1} \in \mathrm{D}^{\mathrm{b}}\left(\mathbf{k}_{M_{12} \times I}\right)$ and $K_{2} \in \mathrm{D}^{\mathrm{b}}\left(\mathbf{k}_{M_{23} \times I}\right)$, we set

$$
\left.K_{1} \circ\right|_{I} K_{2}:=\operatorname{R} q_{13 I !}\left(q_{12 I}^{-1} K_{1} \stackrel{\mathrm{L}}{\otimes} q_{23 I}^{-1} K_{2}\right),
$$

where $q_{i j I}$ is the projection $M_{123} \times I \rightarrow M_{i j} \times I$. The above results extend to the relative case. Namely, we assume the conditions

$$
\left\{\begin{array}{c}
\text { (i) } \operatorname{Supp}\left(K_{1}\right) \times_{M_{2} \times I} \operatorname{Supp}\left(K_{2}\right) \longrightarrow M_{13} \times I \text { is proper, } \\
\text { (ii) } p_{12^{a} I^{a}}^{-1} \Lambda_{1} \cap p_{23 I}^{-1} \Lambda_{2} \cap\left(T_{M_{1}}^{*} M_{1} \times T^{*} M_{2} \times T_{M_{3}}^{*} M_{3} \times T^{*} I\right) \\
\subset T_{M_{1} \times M_{2} \times M_{3} \times I}^{*}\left(M_{1} \times M_{2} \times M_{3} \times I\right),
\end{array}\right.
$$

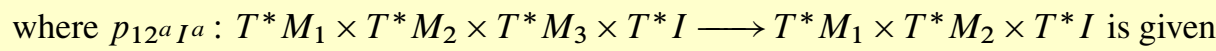
by $p_{12^{a}} I^{a}\left(v_{1}, v_{2}, v_{3}, u\right)=\left(v_{1},-v_{2},-u\right)$. Then we have

$$
\left.\operatorname{SS}\left(\left.K_{1} \circ\right|_{I} K_{2}\right) \subset \Lambda_{1} \circ\right|_{I} \Lambda_{2}:=r_{13}\left(r_{12^{a}}^{-1} \Lambda_{1} \cap r_{23}^{-1} \Lambda_{2}\right) .
$$

Here, in the diagram

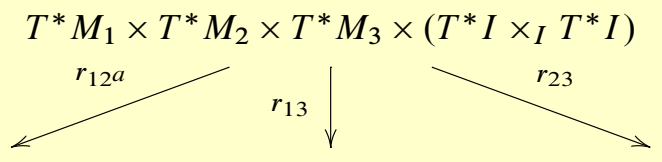

$$
T^{*} M_{1} \times T^{*} M_{2} \times T^{*} I \quad T^{*} M_{1} \times T^{*} M_{3} \times T^{*} I \quad T^{*} M_{2} \times T^{*} M_{3} \times T^{*} I
$$

$r_{12^{a}}$ is given by $p_{12^{a}}: T^{*} M_{1} \times T^{*} M_{2} \times T^{*} M_{3} \rightarrow T^{*} M_{1} \times T^{*} M_{2}$ and the first projection $T^{*} I \times_{I} T^{*} I \rightarrow T^{*} I, r_{23}$ is given by $p_{23}: T^{*} M_{1} \times T^{*} M_{2} \times T^{*} M_{3} \rightarrow$ $T^{*} M_{2} \times T^{*} M_{3}$ and the second projection $T^{*} I \times{ }_{I} T^{*} I \rightarrow T^{*} I$, and $r_{13}$ is given by $p_{13}: T^{*} M_{1} \times T^{*} M_{2} \times T^{*} M_{3} \rightarrow T^{*} M_{1} \times T^{*} M_{3}$ and the addition map $T^{*} I \times I$ $T^{*} I \rightarrow T^{*} I$.

\subsection{Locally bounded categories and gluing sheaves}

Although the prestack $U \mapsto \mathrm{D}\left(\mathbf{k}_{U}\right)$ ( $U$ open in $M$ ) is not a stack, we have the following classical result that we use.

LEMMA 1.11

Let $U_{1}$ and $U_{2}$ be two open subsets of $M$, and set $U_{12}:=U_{1} \cap U_{2}$. Let $F_{i} \in \mathrm{D}\left(\mathbf{k}_{U_{i}}\right)$ $(i=1,2)$, and assume that we have an isomorphism $\varphi_{21}:\left.\left.F_{1}\right|_{U_{12}} \simeq F_{2}\right|_{U_{12}}$. Then 
there exists $F \in \mathrm{D}\left(\mathbf{k}_{U_{1} \cup U_{2}}\right)$ and isomorphisms $\varphi_{i}:\left.F\right|_{U_{i}} \simeq F_{i}(i=1,2)$ such that $\varphi_{12}=\left.\left.\varphi_{2}\right|_{U_{12}} \circ \varphi_{1}\right|_{U_{12}} ^{-1}$. Moreover, such a triple $\left(F, \varphi_{1}, \varphi_{2}\right)$ is unique up to a (nonunique) isomorphism.

Proof

(i) For $*=1,2$ or 12 we let $j_{*}$ be the inclusion of $U_{*}$ in $U_{1} \cup U_{2}$. By adjunction between $j_{12}$ ! and $j_{12}^{-1}$, the morphism $\varphi_{21}$ defines the morphism $u$ : $j_{12 !}\left(\left.F_{1}\right|_{U_{1}}\right) \rightarrow j_{2 !} F_{2}$. We also have the natural morphism $v: j_{12 !}\left(\left.F_{1}\right|_{U_{1}}\right) \rightarrow$ $j_{1} F_{1}$. Then $F$ is given by the distinguished triangle

$$
j_{12 !}\left(\left.F_{1}\right|_{U_{1}}\right) \stackrel{u \oplus v}{\longrightarrow} j_{2 !} F_{2} \oplus j_{1 !} F_{1} \rightarrow F \stackrel{+1}{\longrightarrow} .
$$

(ii) The unicity follows from the distinguished triangle $F_{U_{12}} \rightarrow F_{U_{1}} \oplus F_{U_{2}} \rightarrow$ $F \stackrel{+1}{\longrightarrow}$ and the fact that a commutative square in $\mathrm{D}\left(\mathbf{k}_{M}\right)$ can be extended to a morphism of distinguished triangles.

\section{Definition 1.12}

We say that $F \in \mathrm{D}\left(\mathbf{k}_{M}\right)$ is locally bounded if for any relatively compact open subset $U \subset M$ we have $\left.F\right|_{U} \in \mathrm{D}^{\mathrm{b}}\left(\mathbf{k}_{U}\right)$. We denote by $\mathrm{D}^{\mathrm{lb}}\left(\mathbf{k}_{M}\right)$ the full subcategory of $\mathrm{D}\left(\mathbf{k}_{M}\right)$ consisting of locally bounded objects.

Local notions defined for objects of $\mathrm{D}^{\mathrm{b}}\left(\mathbf{k}_{M}\right)$ extend to objects of $\mathrm{D}^{\mathrm{lb}}\left(\mathbf{k}_{M}\right)$, in particular the microsupport. The Grothendieck operations which preserve boundedness properties also preserve the local boundedness except maybe direct images. However for $F \in \mathrm{D}^{\mathrm{lb}}\left(\mathbf{k}_{M}\right)$ and a morphism of manifolds $f: M \rightarrow N$ which is proper on $\operatorname{Supp}(F)$, we have $\mathrm{R} f_{*} F \simeq \mathrm{R} f_{!} F \in \mathrm{D}^{\mathrm{lb}}\left(\mathbf{k}_{N}\right)$, and Theorem 1.5 still holds in this context.

In particular in the situation of the previous paragraph if we assume that $K_{1} \in$ $\mathrm{D}^{\mathrm{lb}}\left(\mathbf{k}_{M_{12}}\right)$ and $K_{2} \in \mathrm{D}^{\mathrm{lb}}\left(\mathbf{k}_{M_{23}}\right)$ satisfy (1.11), then we obtain $K_{1} \underset{M_{2}}{\circ} K_{2} \in \mathrm{D}^{\mathrm{lb}}\left(\mathbf{k}_{M_{13}}\right)$ with the same bound for its microsupport.

The category $\mathrm{D}^{\mathrm{lb}}\left(\mathbf{k}_{M}\right)$ is stable by the following gluing procedure.

\section{LEMMA 1.13}

Let $j_{n}: U_{n} \hookrightarrow M(n \in \mathbb{N})$ be an increasing sequence of open embeddings of $M$ with $\bigcup_{n} U_{n}=M$. We consider a sequence $\left\{F_{n}\right\}_{n}$ with $F_{n} \in \mathrm{D}^{\mathrm{lb}}\left(\mathbf{k}_{U_{n}}\right)$ together with isomorphisms $u_{n+1, n}:\left.F_{n} \stackrel{\sim}{\longrightarrow} F_{n+1}\right|_{U_{n}}$. Then there exist $F \in \mathrm{D}^{\mathrm{lb}}\left(\mathbf{k}_{M}\right)$ and isomorphisms $u_{n}:\left.F\right|_{U_{n}} \simeq F_{n}$ such that $u_{n+1, n}=u_{n+1} \circ u_{n}^{-1}$ for all $n$. Moreover such $a$ family $\left(F,\left\{u_{n}\right\}_{n}\right)$ is unique up to an (nonunique) isomorphism. 


\section{Proof}

(i) Denote by $v_{n}: j_{n !}\left(F_{n}\right) \rightarrow j_{n+1 !}\left(F_{n+1}\right)$ the morphisms obtained by adjunction. Then define $F \in \mathrm{D}\left(\mathbf{k}_{M}\right)$ as the homotopy colimit of this system; that is, $F$ (which is defined up to isomorphism) is given by the distinguished triangle

$$
\bigoplus_{n \in \mathbb{N}} j_{n !}\left(F_{n}\right) \stackrel{v:=\bigoplus_{n \in \mathbb{N}}\left(\mathrm{id}_{\left.j_{n !}\left(F_{n}\right)-v_{n}\right)}^{\longrightarrow}\right.}{\longrightarrow} \bigoplus_{n \in \mathbb{N}} j_{n !}\left(F_{n}\right) \rightarrow F \stackrel{+1}{\longrightarrow} \text {. }
$$

Then we have isomorphisms $u_{n}:\left.F\right|_{U_{n}} \simeq F_{n}$ for all $n \in \mathbb{N}, u_{n+1, n}=u_{n+1}$ 。 $u_{n}^{-1}$, and $F \in \mathrm{D}^{\mathrm{lb}}\left(\mathbf{k}_{M}\right)$.

(ii) Assume that we have another $G \in \mathrm{D}^{\mathrm{lb}}\left(\mathbf{k}_{M}\right)$ and isomorphisms $w_{n}:\left.G\right|_{U_{n}} \simeq$ $F_{n}$. By adjunction they give $\varphi_{n}: j_{n !}\left(F_{n}\right) \rightarrow G$, and we let $\varphi$ be the sum of the $\varphi_{n}$ 's. Since $u_{n+1, n}=w_{n+1} \circ w_{n}^{-1}$, we have $\varphi \circ v=0$, where $v$ is defined in (1.16). Hence $\varphi$ factorizes through $\psi: F \rightarrow G$. Then $\left.\psi\right|_{U_{n}}=w_{n}^{-1} \circ u_{n}$ is an isomorphism. The property of being an isomorphism being local, we obtain that $\psi$ is an isomorphism.

\subsection{Quantized contact transformations ([13, Section 7.2])}

Consider two manifolds $M$ and $N$, two conic open subsets $U \subset T^{*} M$ and $V \subset T^{*} N$, and a homogeneous contact transformation $\chi$ :

$$
T^{*} N \supset V \underset{\chi}{\stackrel{\sim}{\longrightarrow}} U \subset T^{*} M
$$

Denote by $V^{a}$ the image of $V$ by the antipodal map $a_{N}$ on $T^{*} N$, and denote by $\Lambda$ the image of the graph of $\chi$ by $\operatorname{id}_{U} \times a_{N}$. Hence $\Lambda$ is a conic Lagrangian submanifold of $U \times V^{a}$. A quantized contact transformation (a QCT, for short) above $\chi$ is a kernel $K \in \mathrm{D}^{\mathrm{b}}\left(\mathbf{k}_{M \times N}\right)$ such that $\mathrm{SS}(K) \cap\left(U \times V^{a}\right)=\Lambda$ and satisfying some technical properties that we do not recall here so that the kernel $K$ induces an equivalence of categories

$$
K \circ \cdot: \mathrm{D}^{\mathrm{b}}\left(\mathbf{k}_{N} ; V\right) \stackrel{\sim}{\longrightarrow} \mathrm{D}^{\mathrm{b}}\left(\mathbf{k}_{M} ; U\right) .
$$

Given $\chi$ and $q \in V, p=\chi(q) \in U$, there exists such a QCT after replacing $U$ and $V$ by sufficiently small neighborhoods of $p$ and $q$.

\subsection{The functor $\mu$ hom ([13, Sections 4.4, 7.2])}

The functor of microlocalization along a submanifold was introduced by Mikio Sato in the 1970 s and has been at the origin of what is now called microlocal analysis. A variant of this functor, the bifunctor

$$
\text { بhom: } \mathrm{D}^{\mathrm{b}}\left(\mathbf{k}_{M}\right)^{\mathrm{op}} \times \mathrm{D}^{\mathrm{b}}\left(\mathbf{k}_{M}\right) \rightarrow \mathrm{D}^{\mathrm{b}}\left(\mathbf{k}_{T^{*} M}\right)
$$


has been constructed in [13]. Since $\operatorname{Supp}\left(\mu h o m\left(F, F^{\prime}\right)\right) \subset \operatorname{SS}(F) \cap \operatorname{SS}\left(F^{\prime}\right)$, (1.19) induces a bifunctor for any open subset $U$ of $T^{*} M$ :

$$
\text { uhom: } \mathrm{D}^{\mathrm{b}}\left(\mathbf{k}_{M} ; U\right)^{\mathrm{op}} \times \mathrm{D}^{\mathrm{b}}\left(\mathbf{k}_{M} ; U\right) \rightarrow \mathrm{D}^{\mathrm{b}}\left(\mathbf{k}_{U}\right) .
$$

Let us only recall the properties of this functor that we use. Consider a function $\psi: M \rightarrow \mathbb{R}$ defined in a neighborhood $W$ of $x_{0} \in M$ such that $d \psi\left(x_{0}\right) \neq 0$. Then, setting $S:=\left\{x \in W ; \psi(x)=\psi\left(x_{0}\right)\right\}$ and $p=d \psi\left(x_{0}\right)$, we have

$$
\mathrm{R} \Gamma_{\left\{\psi(x) \geq \psi\left(x_{0}\right)\right\}}(F)_{x_{0}} \simeq \mu h o m\left(\mathbf{k}_{S}, F\right)_{p} \quad \text { for any } F \in \mathrm{D}^{\mathrm{b}}\left(\mathbf{k}_{M}\right) .
$$

If $\chi$ is a contact transform as in (1.17) and if $K$ is a QCT as in (1.18), then $K$ induces a natural isomorphism for any $F, G \in \mathrm{D}^{\mathrm{b}}\left(\mathbf{k}_{N} ; V\right)$

$$
\left.\chi_{*}\left(\left.\mu \operatorname{hom}(F, G)\right|_{V}\right) \stackrel{\sim}{\longrightarrow} \mu \operatorname{hom}(K \circ F, K \circ G)\right|_{U} .
$$

\subsection{Simple sheaves ([13, Section 7.5])}

Let $\Lambda \subset \dot{T}^{*} M$ be a locally closed conic Lagrangian submanifold, and let $p \in \Lambda$. Simple sheaves along $\Lambda$ at $p$ are defined in [13, Definition 7.5.4].

When $\Lambda$ is the conormal bundle to a submanifold $N \subset M$, that is, when the projection $\left.\pi_{M}\right|_{\Lambda}: \Lambda \rightarrow M$ has constant rank, then an object $F \in \mathrm{D}^{\mathrm{b}}\left(\mathbf{k}_{M}\right)$ is simple along $\Lambda$ at $p$ if $F \simeq \mathbf{k}_{N}[d]$ in $\mathrm{D}^{\mathrm{b}}\left(\mathbf{k}_{M} ; p\right)$ for some shift $d \in \mathbb{Z}$.

If $\operatorname{SS}(F)$ is contained in $\Lambda$ on a neighborhood of $\Lambda, \Lambda$ is connected and $F$ is simple at some point of $\Lambda$, then $F$ is simple at every point of $\Lambda$.

If $\Lambda_{1} \subset T^{*} M_{12}$ and $\Lambda_{2} \subset T^{*} M_{23}$ are locally closed conic Lagrangian submanifolds and if $K_{i} \in \mathrm{D}^{\mathrm{b}}\left(\mathbf{k}_{M_{i, i+1}}\right)(i=1,2)$ are simple along $\Lambda_{i}$, then $K_{1} \circ K_{2}$ is simple along $\Lambda_{1} \circ \Lambda_{2}$ under some conditions (see [13, Theorem 7.5.11]). In particular, simple sheaves are stable by QCT.

Now, let $M$ and $N$ be two manifolds with the same dimension. Let $F \in$ $\mathrm{D}^{\mathrm{b}}\left(\mathbf{k}_{M \times N}\right)$. Set

$$
F^{-1}=v^{-1} \mathrm{R} \mathscr{H o m}\left(F, \omega_{M} \otimes \mathbf{k}_{N}\right) \in \mathrm{D}^{\mathrm{b}}\left(\mathbf{k}_{N \times M}\right),
$$

where $v: N \times M \rightarrow M \times N$ is the swap. Let $q_{i j}$ be the $(i, j)$ th projection from $N \times$ $M \times N$. Then we have $F^{-1} \circ F=\operatorname{R} q_{13 !}\left(q_{12}^{-1} F^{-1} \stackrel{\mathrm{L}}{\otimes} q_{23}^{-1} F\right)$. Let $\delta: N \rightarrow N \times N$ be the diagonal embedding. Then we have $\delta^{-1}\left(F^{-1} \circ F\right) \simeq \mathrm{R} q_{2 !}\left(F \stackrel{\mathrm{L}}{\otimes} \mathrm{R} \mathscr{H o m}\left(F, \omega_{M} \otimes\right.\right.$ $\left.\left.\mathbf{k}_{N}\right)\right)$. Hence $\delta^{-1}\left(F^{-1} \circ F\right) \simeq \mathrm{R} q_{2 !}\left(F \stackrel{\mathrm{L}}{\otimes} \mathrm{R} \mathscr{H o m}\left(F, q_{2}^{!} \mathbf{k}_{N}\right)\right) \rightarrow \operatorname{R} q_{2 !}\left(q_{2}^{!} \mathbf{k}_{N}\right) \rightarrow \mathbf{k}_{N}$ gives a morphism

$$
F^{-1} \circ F \rightarrow \mathbf{k}_{\Delta_{N}}
$$


PROPOSITION 1.14 ([13, Propositions 7.1.8, 7.1.9, Theorem 7.2.1])

Let $F \in \mathrm{D}^{\mathrm{b}}\left(\mathbf{k}_{M \times N}\right)$, let $p_{M} \in \dot{T}^{*} M$, and let $p_{N} \in \dot{T}^{*} N$. Assume the following conditions:

(i) $\operatorname{Supp}(F) \rightarrow N$ is proper,

(ii) $\quad F$ is cohomologically constructible (see [13, Definition 3.4.1]),

(iii) $\operatorname{SS}(F) \cap\left(\dot{T}^{*} M \times T_{N}^{*} N\right)=\emptyset$,

(iv) $\operatorname{SS}(F) \cap\left(T^{*} M \times\left\{p_{N}^{a}\right\}\right)=\left\{\left(p_{M}, p_{N}^{a}\right)\right\}$,

(v) $\mathrm{SS}(F)$ is a Lagrangian submanifold of $T^{*}(M \times N)$ on a neighborhood of $\left(p_{M}, p_{N}^{a}\right)$,

(vi) $\quad F$ is simple along $\mathrm{SS}(F)$ at $\left(p_{M}, p_{N}^{a}\right)$,

(vii) $\mathrm{SS}(F) \rightarrow T^{*} N$ is a local isomorphism at $\left(p_{M}, p_{N}^{a}\right)$.

Then the morphism $F^{-1} \circ F \rightarrow \mathbf{k}_{\Delta_{N}}$ is an isomorphism in $\mathrm{D}^{\mathrm{b}}\left(\mathbf{k}_{N \times N} ;\left(p_{N}, p_{N}^{a}\right)\right)$.

\section{Deformation of the conormal to the diagonal}

As usual, we denote by $\Delta_{M}$ or simply $\Delta$ the diagonal of $M \times M$. We denote by $p_{1}$ and $p_{2}$ the first and second projections from $T^{*}(M \times M)$ to $T^{*} M$ and by $p_{2}^{a}$ the composition of $p_{2}$ and the antipodal map on $T^{*} M$. We also set $n:=\operatorname{dim} M$.

Consider a $\mathrm{C}^{\infty}$-function $f(x, y)$ defined on an open neighborhood $\Omega_{0} \subset M \times M$ of the diagonal $\Delta_{M}$. We assume that

(i) $\left.f\right|_{\Delta_{M}} \equiv 0$,

(ii) $f(x, y)>0$ for $(x, y) \in \Omega_{0} \backslash \Delta_{M}$,

(iii) the partial Hessian $\frac{\partial^{2} f}{\partial x_{i} \partial x_{j}}(x, y)$ is positive definite for any $(x, y) \in \Delta_{M}$.

Such a pair $\left(\Omega_{0}, f\right)$ exists.

PROPOSITION 2.1

Assume that $\left(\Omega_{0}, f\right)$ satisfies the conditions (i)-(iii) above. Let $U$ be a relatively compact open subset of $M$. Then there exist an $\varepsilon>0$ and an open subset $\Omega$ of $M \times M$ satisfying the following conditions:

(a) $\Delta_{U} \subset \Omega \subset \Omega_{0} \cap(M \times U)$,

(b) $Z_{\varepsilon}:=\{(x, y) \in \Omega ; f(x, y) \leq \varepsilon\}$ is proper over $U$ by the map induced by the second projection,

(c) for any $y \in U$ and $\left.\left.\varepsilon^{\prime} \in\right] 0, \varepsilon\right]$, the open subset $\{x \in M ;(x, y) \in \Omega, f(x, y)<$ $\varepsilon^{\prime}$ \} is homeomorphic to $\mathbb{R}^{n}$,

(d) $d_{x} f(x, y) \neq 0, d_{y} f(x, y) \neq 0$ for $(x, y) \in \Omega \backslash \Delta_{M}$,

(e) setting $\Gamma_{Z_{\varepsilon}}=\left\{(x, y ; \xi, \eta) \in T^{*}(\Omega) ; f(x, y)=\varepsilon,(\xi, \eta)=\lambda d f(x, y), \lambda<0\right\}$, the projection $p_{2}^{a}: T^{*}(M \times U) \rightarrow T^{*} U$ induces an isomorphism $\Gamma_{Z_{\varepsilon}} \underset{p_{2}^{a}}{\sim}$ $\dot{T}^{*} U$ and the projection $p_{1}: T^{*}(M \times U) \rightarrow T^{*} M$ induces an open embed$\operatorname{ding} \Gamma_{Z_{\varepsilon}} \hookrightarrow \dot{T}^{*} M$. 
Proof

Replacing $\Omega_{0}$ with the open subset

$$
\Delta_{M} \cup\left\{(x, y) \in \Omega_{0} ; d_{x} f(x, y) \neq 0, d_{y} f(x, y) \neq 0\right\},
$$

we may assume from the beginning that $\Omega_{0}$ satisfies $(d)$.

Let $F: \Omega_{0} \rightarrow T^{*} M$ be the map $(x, y) \mapsto d_{y} f(x, y)$. This map sends $\Delta_{M}$ to $T_{M}^{*} M$, and is a local isomorphism. Then there exists an open neighborhood $\Omega^{\prime} \subset \Omega_{0}$ of $\Delta_{M}$ such that $\left.F\right|_{\Omega^{\prime}}: \Omega^{\prime} \rightarrow T^{*} M$ is an open embedding. Hence by identifying $\Omega^{\prime}$ as its image, we can reduce the proposition to the following lemma.

\section{LEMMA 2.2}

Let $p: E \rightarrow X$ be a vector bundle of rank $n$, let $i: X \rightarrow E$ be the zero-section, let $S E=(E \backslash i(X)) / \mathbb{R}_{>0}$ be the associated sphere bundle, and let $q: E \backslash i(X) \rightarrow S E$ be the projection. Let $f$ be a $\mathrm{C}^{\infty}$-function on a neighborhood $\Omega$ of the zero-section $i(X)$ of $E$. Assume the following conditions:

(i) $f(z)=0$ for $z \in i(X)$,

(ii) $f(z)>0$ for $z \in \Omega \backslash i(X)$,

(iii) for any $x \in X$ the Hessian of $\left.f\right|_{p^{-1}(x)}$ at $i(x)$ is positive-definite.

Then, for any relatively compact open subset $U$ of $X$, there exist $\varepsilon>0$ and an open subset $\Omega^{\prime} \subset \Omega \cap p^{-1}(U)$ containing $i(U)$ that satisfy the following conditions:

(a) $\left\{z \in \Omega^{\prime} ; f(z) \leq \varepsilon\right\}$ is proper over $U$,

(b) $\left.\quad\left\{z \in \Omega^{\prime} ; 0<f(z)<\varepsilon\right\} \rightarrow\left(S E \times_{X} U\right) \times\right] 0, \varepsilon[$ given by $z \mapsto(q(z), f(z))$ is an isomorphism,

(c) for any $x \in U$ and $t \in] 0$, $\varepsilon\left[\right.$, the set $\left\{z \in \Omega^{\prime} \cap p^{-1}(x) ; f(z)<t\right\}$ is homeomorphic to $\mathbb{R}^{n}$.

Since the proof is elementary, we omit it.

Recall (1.21).

\section{THEOREM 2.3}

We keep the notation in Proposition 2.1 and set $L=\mathbf{k}_{Z_{\varepsilon}} \in \mathrm{D}^{\mathrm{b}}\left(\mathbf{k}_{M \times U}\right)$. Then $\mathrm{SS}(L) \subset \Gamma_{Z_{\varepsilon}} \cup Z_{\varepsilon}$ and $L^{-1} \circ L \stackrel{\sim}{\longrightarrow} \mathbf{k}_{\Delta_{U}}$.

\section{Proof}

Set $Z=Z_{\varepsilon}$. We have $\operatorname{SS}\left(L^{-1} \circ L\right) \subset T_{\Delta_{U}}^{*}(U \times U) \cup T_{U \times U}^{*}(U \times U)$. By Proposition 1.14, there exists a morphism $L^{-1} \circ L \rightarrow \mathbf{k}_{\Delta_{U}}$ which is an isomorphism in $\mathrm{D}^{\mathrm{b}}\left(\mathbf{k}_{U \times U} ; \dot{T}^{*}(U \times U)\right)$. Hence if $N \rightarrow L^{-1} \circ L \rightarrow \mathbf{k}_{\Delta_{U}} \stackrel{+1}{\longrightarrow}$ is a distinguished triangle, then $\operatorname{SS}(N) \subset T_{U \times U}^{*}(U \times U)$, and hence $N$ has locally constant cohomologies. 
In particular $\operatorname{Supp}(N)$ is open and closed in $U \times U$. Let $\delta: U \rightarrow U \times U$ be the diagonal embedding. Then we have $\delta^{-1}\left(L^{-1} \circ L\right) \simeq \mathrm{R} q_{2 !}\left(L \stackrel{\mathrm{L}}{\otimes} \mathrm{R} \mathscr{H} \circ m\left(L, \mathbf{k}_{M \times U}\right) \stackrel{\mathrm{L}}{\otimes}\right.$ $\left.q_{2}^{!} \mathbf{k}_{U}\right)$. Since $L \simeq \mathbf{k}_{Z}$ and $\mathrm{R} \mathscr{H o m}\left(L, \mathbf{k}_{M \times U}\right) \simeq \mathbf{k}_{\operatorname{Int}(Z)}$, we have $\delta^{-1}\left(L^{-1} \circ L\right) \simeq$ $\mathrm{R} q_{2 !}\left(\mathbf{k}_{\text {Int }(Z)} \stackrel{\mathrm{L}}{\otimes} q_{2}^{!} \mathbf{k}_{U}\right)$. Since the fibers of $\operatorname{Int}(Z) \rightarrow U$ are homeomorphic to $\mathbb{R}^{n}$, we have $\mathrm{R} q_{2 !}\left(\mathbf{k}_{\text {Int }(Z)} \stackrel{\mathrm{L}}{\otimes} q_{2}^{!} \mathbf{k}_{U}\right) \simeq \mathbf{k}_{U}$. Thus we obtain $\delta^{-1}\left(L^{-1} \circ L\right) \simeq \mathbf{k}_{U}$ and hence $\delta^{-1} N \simeq 0$. Hence $\operatorname{Supp}(N) \cap \Delta_{U}=\emptyset$ and $\operatorname{Supp}(N) \subset \operatorname{Supp}\left(L^{-1} \circ L\right)$. Since we have

$$
\operatorname{Supp}\left(L^{-1} \circ L\right) \subset\left\{\left(y, y^{\prime}\right) \in U \times U ;(x, y),\left(x, y^{\prime}\right) \in Z \text { for some } x \in M\right\}
$$

and the fiber of $Z \rightarrow U$ is connected, $y$ and $y^{\prime}$ belong to the same connected component of $M$ as soon as $\left(y, y^{\prime}\right) \in \operatorname{Supp}(N)$. Since $\operatorname{Supp}(N)$ is open and closed in $U \times U$ and $\operatorname{Supp}(N) \cap \Delta_{U}=\emptyset$, we conclude that $\operatorname{Supp}(N)=\emptyset$.

\section{Quantization of homogeneous Hamiltonian isotopies}

Let $M$ be a real manifold of class $\mathrm{C}^{\infty}$, and let $I$ be an open interval of $\mathbb{R}$ containing the origin. We consider a $C^{\infty}$-map $\Phi: \dot{T}^{*} M \times I \rightarrow \dot{T}^{*} M$. Setting $\varphi_{t}=\Phi(\bullet, t)$ $(t \in I)$, we always assume

$$
\left\{\begin{array}{l}
\varphi_{t} \text { is a homogeneous symplectic isomorphism for each } t \in I, \\
\varphi_{0}=\mathrm{id}_{\dot{T}^{*} M}
\end{array}\right.
$$

Let us recall here some classical facts that we explain with more details in the appendix. Set

$$
\begin{aligned}
& v_{\Phi}:=\frac{\partial \Phi}{\partial t}: \dot{T}^{*} M \times I \rightarrow T \dot{T}^{*} M, \\
& f=\left\langle\alpha_{M}, v_{\Phi}\right\rangle: \dot{T}^{*} M \times I \rightarrow \mathbb{R}, \quad f_{t}=f(\cdot, t) .
\end{aligned}
$$

Denote by $H_{g}$ the Hamiltonian flow of a function $g: \dot{T}^{*} M \rightarrow \mathbb{R}$. Then

$$
\frac{\partial \Phi}{\partial t}=H_{f_{t}} .
$$

In other words, $\Phi$ is a homogeneous Hamiltonian isotopy.

In this situation, there exists a unique conic Lagrangian submanifold $\Lambda$ of $\dot{T}^{*} M \times$ $\dot{T}^{*} M \times T^{*} I$ closed in $\dot{T}^{*}(M \times M \times I)$ such that, setting

$$
\Lambda_{t}=\Lambda \circ T_{t}^{*} I
$$

$\Lambda_{t}$ is the graph of $\varphi_{t}$ (see Lemma A.2).

The main result of this section is the existence and unicity of an object $K \in$ $\mathrm{D}^{\mathrm{lb}}\left(\mathbf{k}_{M \times M \times I}\right)$ whose microsupport is contained in $\Lambda$ outside the zero-section and 
whose restriction at $t=0$ is $\mathbf{k}_{\Delta}$. We call $K$ the quantization of $\Phi$ on $I$ or of $\left\{\varphi_{t}\right\}_{t \in I}$. We first prove that if such a $K$ exists, then its support has some properness properties from which we deduce its unicity. Then we prove the existence assuming that

$$
\left\{\begin{array}{l}
\text { there exists a compact subset } A \text { of } M \text { such that } \varphi_{t} \text { is the identity } \\
\text { outside of } \dot{\pi}_{M}^{-1}(A) \text { for all } t \in I, \dot{\pi}_{M}: \dot{T}^{*} M \rightarrow M \text { denoting the } \\
\text { projection. }
\end{array}\right.
$$

For this purpose we glue local constructions by using the unicity. Then we prove the existence in general, using an approximation of $\Phi$ by Hamiltonian isotopies satisfying (3.3).

\subsection{Unicity and support of the quantization}

We introduce the following notations:

$$
\begin{aligned}
& I_{t}=[0, t] \text { or }[t, 0] \text { according to the sign of } t \in I, \\
& B:=\left\{(x, y, t) \in M \times M \times I ;\left(\{x\} \times\{y\} \times I_{t}\right) \cap \dot{\pi}_{M \times M \times I}(\Lambda) \neq \emptyset\right\} .
\end{aligned}
$$

LEMMA 3.1

Both projections $B \rightrightarrows M \times I$ are proper.

\section{Proof}

(i) Let us show that the second projection $q: B \rightarrow M \times I$ is proper. We see easily that $q^{-1}(y, t)=\dot{\pi}_{M}\left(\Phi\left(\dot{\pi}_{M}^{-1}(y) \times I_{t}\right)\right) \times\{y\} \times\{t\}$. We choose a compact set $D \subset M$ and $t \in I$. Then $q^{-1}\left(D \times I_{t}\right)$ is contained in $\dot{\pi}_{M}\left(\Phi\left(\dot{\pi}_{M}^{-1}(D) \times I_{t}\right)\right) \times$ $D \times I_{t}$ which is compact.

(ii) The first projection is treated similarly by reversing the roles of $x$ and $y$ and replacing $\Phi$ by $\Phi^{-1}=\left\{\varphi_{t}^{-1}\right\}_{t \in I}$.

Recall that for $F \in \mathrm{D}^{\mathrm{lb}}\left(\mathbf{k}_{M \times N}\right)$, the object $F^{-1}$ is defined in (1.21). For an object $K \in \mathrm{D}^{\mathrm{lb}}\left(\mathbf{k}_{M \times M \times I}\right)$ and $t_{0} \in I$, we set

$$
K_{t_{0}}=\left.K\right|_{t=t_{0}} \simeq K \circ \mathbf{k}_{t=t_{0}} \in \mathrm{D}^{\mathrm{lb}}\left(\mathbf{k}_{M \times M}\right) .
$$

We also set (keeping the same notation for $v$ as in (1.21)):

$$
K^{-1}=\left(v \times \operatorname{id}_{I}\right)^{-1} \mathrm{R} \mathscr{H o m}\left(K, \omega_{M} \otimes \mathbf{k}_{M} \otimes \mathbf{k}_{I}\right) .
$$

Then, assuming

$$
\operatorname{SS}(K) \cap T_{M \times M}^{*}(M \times M) \times T^{*} I \subset T_{M \times M \times I}^{*}(M \times M \times I),
$$


we have $\left(K^{-1}\right)_{t} \simeq\left(K_{t}\right)^{-1}$ for any $t \in I$ (see the proof of (ii) in the proposition below).

PROPOSITION 3.2

We assume that $\Phi$ satisfies hypothesis (3.1) and that $K \in \mathrm{D}^{\mathrm{lb}}\left(\mathbf{k}_{M \times M \times I}\right)$ satisfies the following conditions:

(a) $\operatorname{SS}(K) \subset \Lambda \cup T_{M \times M \times I}^{*}(M \times M \times I)$,

(b) $K_{0} \simeq \mathbf{k}_{\Delta}$.

Then we have

(i) $\operatorname{Supp}(K) \subset B$ (see (3.4)), and both projections $\operatorname{Supp}(K) \rightrightarrows M \times I$ are proper,

(ii) $K_{t} \circ K_{t}^{-1} \simeq K_{t}^{-1} \circ K_{t} \simeq \mathbf{k}_{\Delta}$ for all $t \in I$,

(iii) such a $K$ satisfying the conditions $(a)$ and $(b)$ is unique up to a unique isomorphism,

(iv) if there exists an open set $W \subset M$ such that $\left.\varphi_{t}\right|_{\dot{\pi}_{M}^{-1}(W)}=\mathrm{id}$ for all $t \in I$, then $\left.\left.K\right|_{(W \times M \cup M \times W) \times I} \simeq \mathbf{k}_{\Delta \times I}\right|_{(W \times M \cup M \times W) \times I}$.

Proof

(i) Let us prove (i). Since $\Lambda$ is closed and conic, $\dot{\pi}_{M \times M \times I}(\Lambda)$ is closed. So if $(x, y, t) \notin B$ we may find open connected neighborhoods $U$ of $x, V$ of $y$, and $J$ of $I_{t}$ such that $\dot{\pi}_{M \times M \times I}^{-1}(U \times V \times J)$ does not meet $\Lambda$. By condition (a) this implies that $\operatorname{SS}\left(\left.K\right|_{U \times V \times J}\right)$ is contained in the zero-section. Hence $K$ is locally constant on $U \times V \times J$. Now $0 \in J$, and $U \times V$ does not meet $\Delta_{M}$ since $\dot{\pi}_{M \times M \times I}(\Lambda)$ contains $\Delta_{M} \times\{0\}$. Hence $\left.K\right|_{U \times V \times\{0\}}=0$, and we deduce $\left.K\right|_{U \times V \times J}=0$. In particular $(x, y, t) \notin \operatorname{Supp}(K)$, and this proves $\operatorname{Supp}(K) \subset B$. To conclude, we apply Lemma 3.1.

(ii) Let us prove (ii). We set $F=\left.K \circ\right|_{I} K^{-1}$ (see notation (1.13)). Hence (ii) is implied by $F \simeq \mathbf{k}_{\Delta \times I}$. Let $v$ be the involution of $T^{*} M \times T^{*} M \times T^{*} I$ given by $v\left(x, \xi, x^{\prime}, \xi^{\prime}, t, \tau\right)=\left(x^{\prime},-\xi^{\prime}, x,-\xi, t,-\tau\right)$. Then we have

$$
\mathrm{SS}\left(K^{-1}\right) \cap \dot{T}^{*}(M \times M \times I) \subset v(\Lambda) .
$$

Hence by (1.15), $\mathrm{SS}(F)$ satisfies

$$
\begin{aligned}
\mathrm{SS}(F) & \subset T_{\Delta_{M \times I}^{*}}^{*}(M \times M \times I) \cup T_{M \times M \times I}^{*}(M \times M \times I) \\
& \subset T^{*}(M \times M) \times T_{I}^{*} I .
\end{aligned}
$$

By Corollary 1.6, $F$ is constant on the fibers of $M \times M \times I \rightarrow M \times M$. Denote by $i_{0}: M \times M \rightarrow M \times M \times I$ the inclusion associated to $\{t=0\} \subset I$. It is thus enough to prove the isomorphism $i_{0}^{-1} F \simeq \mathbf{k}_{\Delta}$. We have 


$$
\begin{aligned}
i_{0}^{!} \mathrm{R} \mathscr{H o m}\left(K, \mathbf{k}_{M \times M \times I}\right) & \simeq \mathrm{R} \mathscr{H o m}\left(i_{0}^{-1} K, i_{0}^{!} \mathbf{k}_{M \times M \times I}\right) \\
& \simeq \mathrm{R} \mathscr{H o m}\left(K_{0}, \mathbf{k}_{M \times M}\right) \stackrel{\mathrm{L}}{\otimes} i_{0}^{!} \mathbf{k}_{M \times M \times I} .
\end{aligned}
$$

On the other hand, the condition on $\operatorname{SS}(K)$ implies

$$
i_{0}^{!} \mathrm{R} \mathscr{H o m}\left(K, \mathbf{k}_{M \times M \times I}\right) \simeq i_{0}^{-1} \mathrm{R} \mathscr{H o m}\left(K, \mathbf{k}_{M \times M \times I}\right) \stackrel{\mathrm{L}}{\otimes} i_{0}^{!} \mathbf{k}_{M \times M \times I} .
$$

Therefore $i_{0}^{-1} \mathrm{R} \mathscr{H o m}\left(K, \mathbf{k}_{M \times M \times I}\right) \simeq \mathrm{R} \mathscr{H o m}\left(K_{0}, \mathbf{k}_{M \times M}\right)$ which gives the isomorphism $i_{0}^{-1} K^{-1} \simeq K_{0}^{-1}$. Thus we obtain $i_{0}^{-1} F \simeq K_{0} \circ K_{0}^{-1} \simeq \mathbf{k}_{\Delta}$ as required.

(iii) This is a particular case of the more precise Lemma 3.3 below.

(iv) We set $\widetilde{W}=(W \times M \cup M \times W) \times I$. Then $B \cap \widetilde{W}=\Delta_{W} \times I$. Hence (i) implies that $\operatorname{Supp}(K) \cap \widetilde{W} \subset \Delta_{W} \times I$. Then (b) implies (iv).

LEMMA 3.3

Let $\Phi_{i}: \dot{T}^{*} M \times I \rightarrow \dot{T}^{*} M(i=1,2)$ be two maps satisfying (3.1), and define $\Lambda_{i} \subset$ $\dot{T}^{*} M \times \dot{T}^{*} M \times T^{*} I$ as in Lemma A.2. Assume that there exist $K_{i} \in \mathrm{D}^{\mathrm{lb}}\left(\mathbf{k}_{M \times M \times I}\right)$ $(i=1,2)$ satisfying conditions ( $a)$ and (b) of Proposition 3.2. Also assume that there exists an open set $U \subset M$ such that

$$
\left.\Phi_{1}\right|_{\dot{\pi}_{M}^{-1}(U) \times I}=\left.\Phi_{2}\right|_{\dot{\pi}_{M}^{-1}(U) \times I}
$$

Then there exists a unique isomorphism $\psi:\left.\left.K_{1}\right|_{M \times U \times I} \stackrel{\sim}{\longrightarrow} K_{2}\right|_{M \times U \times I}$ such that

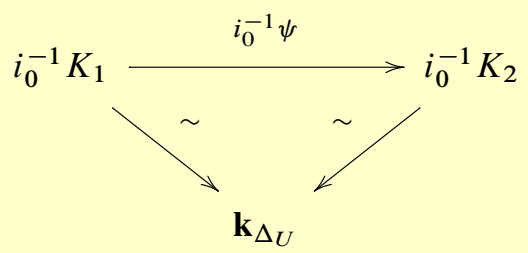

commutes, where $i_{0}: M \times U \rightarrow M \times U \times I$ is the inclusion by $0 \in I$.

\section{Proof}

We define $\Phi_{2}^{-1}: \dot{T}^{*} M \times I \rightarrow \dot{T}^{*} M$ by $\varphi_{2, t}^{-1}=\left(\varphi_{2, t}\right)^{-1}$ for all $t \in I$. Then, similarly to (3.5), we have $\operatorname{SS}\left(K_{2}^{-1}\right) \subset v\left(\Lambda_{2}\right)$ outside the zero-section. We also define $\Phi: \dot{T}^{*} M \times I \rightarrow \dot{T}^{*} M$ by $\varphi_{t}=\varphi_{2, t}^{-1} \circ \varphi_{1, t}$. Its associated Lagrangian submanifold is $\Lambda=\left.v\left(\Lambda_{2}\right) \circ\right|_{I} \Lambda_{1}$ (see (1.15)). By (3.6) we have $\varphi_{t}(x, \xi)=(x, \xi)$ for all $t \in I$ and $(x, \xi) \in \dot{\pi}_{M}^{-1}(U)$. Hence

$$
\Lambda \cap \dot{T}^{*}(M \times U \times I)=\dot{T}_{\Delta_{U}}^{*}(M \times U) \times T_{I}^{*} I .
$$


We set $L=\left.K_{2}^{-1} \circ\right|_{I} K_{1}$. By (1.15), we have the inclusion $\operatorname{SS}(L) \subset \Lambda$ outside the zero-section. It follows that $\mathrm{SS}(L)$ is contained in $T^{*}(M \times U) \times T_{I}^{*} I$ over $M \times U \times I$. Since $L_{0} \simeq \mathbf{k}_{\Delta_{M}}$, we deduce from Corollary 1.6 that $\left.L\right|_{M \times U \times I} \simeq \mathbf{k}_{\left(\Delta_{M} \cap M \times U\right) \times I}$. Then

$$
\left.\left.\left.\left.K_{1}\right|_{M \times U \times I} \simeq\left(\left.K_{2} \circ\right|_{I} L\right)\right|_{M \times U \times I} \simeq K_{2} \circ\right|_{I}\left(\left.L\right|_{M \times U \times I}\right) \simeq K_{2}\right|_{M \times U \times I}
$$

as claimed.

The uniqueness of $\psi$ follows from the uniqueness of the isomorphism $\left.L\right|_{M \times U \times I} \simeq \mathbf{k}_{\Delta_{U} \times I}$.

\subsection{Existence of the quantization: "Compact" case}

Lemma 3.5 below is the main step in the proof of Theorem 3.7. We prove the existence of a quantization of a homogeneous Hamiltonian isotopy $\Phi: \dot{T}^{*} M \times I \rightarrow \dot{T}^{*} M$ satisfying hypotheses (3.1) and (3.3) (i.e., $\varphi_{t}$ is the identity map outside $\dot{\pi}_{M}^{-1}(A)$ for each $t \in I$, where $A \subset M$ is compact). In the course of the proof we need an elementary lemma that we state without proof.

\section{LEMMA 3.4}

Let $N$ be a manifold, let $V_{0} \subset N$ be an open subset with a smooth boundary, let $C \subset N$ be a compact subset, and let I be an open interval of $\mathbb{R}$ containing zero. Let $\Lambda \subset \dot{T}^{*}(N \times I)$ be a closed conic Lagrangian submanifold, and set $\Lambda_{t}=\Lambda \circ T_{t}^{*} I$ for $t \in I$. We assume that$$
\Lambda_{0}=\operatorname{SS}\left(\mathbf{k}_{\overline{V_{0}}}\right) \cap \dot{T}^{*} N \text {, }
$$$$
\text { (c) } \quad \Lambda \subset \dot{T}^{*} N \times T^{*} I \text { and } \Lambda \rightarrow \dot{T}^{*} N \times I \text { is a closed embedding. }
$$

Then there exist $\varepsilon>0$ with $\pm \varepsilon \in I$ and an open subset $V \subset N \times]-\varepsilon, \varepsilon$ [ with a smooth boundary such that

(i) $\quad V_{0}=V \cap(N \times\{0\})$,

(ii) $\quad \Lambda=\operatorname{SS}\left(\mathbf{k}_{\bar{V}}\right) \cap \dot{T}^{*}(N \times]-\varepsilon, \varepsilon[)$,

(iii) $\quad \Lambda_{t}=\operatorname{SS}\left(\mathbf{k}_{\overline{V \cap(N \times\{t\})}}\right) \cap \dot{T}^{*} N$ for any $\left.t \in\right]-\varepsilon, \varepsilon[$.

\section{LEMMA 3.5}

Assume that $\Phi$ satisfies hypotheses (3.1) and (3.3). Then there exists $K \in$ $\mathrm{D}^{\mathrm{lb}}\left(\mathbf{k}_{\boldsymbol{M} \times M \times I}\right)$ satisfying conditions (a) and (b) of Proposition 3.2.

\section{Proof}

(A) Local existence. We first prove that there exists $\varepsilon>0$ such that there exists a quantization $\widetilde{K} \in \mathrm{D}^{\mathrm{b}}\left(\mathbf{k}_{M \times M \times]-\varepsilon, \varepsilon[}\right)$ of $\Phi$ on $]-\varepsilon, \varepsilon[$. 
We use the results and notation of Proposition 2.1 and Theorem 2.3. We choose a relatively compact open subset $U$ such that $A \subset U \subset M$, where $A$ is given in hypothesis (3.3). We choose $f$ and $\varepsilon_{1}$ as in Proposition 2.1 (in which $\varepsilon_{1}$ was denoted by $\varepsilon$ ). Then $L:=\mathbf{k}_{Z_{\varepsilon_{1}}} \in \mathrm{D}^{\mathrm{b}}\left(\mathbf{k}_{M \times U}\right)$ satisfies $\operatorname{SS}(L)=\Gamma_{Z_{\varepsilon_{1}}} \cup Z_{\varepsilon_{1}}$, and $L^{-1} \circ L \simeq \mathbf{k}_{\Delta_{U}}$. We define for $t \in I$

$$
\begin{aligned}
& \widetilde{\Lambda}:=\Gamma_{Z_{\varepsilon_{1}}} \circ \Lambda \subset \dot{T}^{*} M \times \dot{T}^{*} U \times T^{*} I, \\
& \widetilde{\Lambda}_{t}:=\Gamma_{Z_{\varepsilon_{1}}} \circ \Lambda_{t} \subset \dot{T}^{*} M \times \dot{T}^{*} U .
\end{aligned}
$$

We remark that $\widetilde{\Lambda}_{t}=\widetilde{\Lambda} \circ T_{t}^{*} I$ and $\widetilde{\Lambda}_{0}=\operatorname{SS}\left(\mathbf{k}_{Z_{\varepsilon_{1}}}\right) \cap \dot{T}^{*}(U \times I)$. We apply Lemma 3.4 with $N=M \times U, C=A \times A, V_{0}=\operatorname{Int} Z_{\varepsilon_{1}}$. We obtain $\varepsilon>0$ and an open subset $V \subset M \times U \times]-\varepsilon, \varepsilon\left[\right.$ such that $\widetilde{L}:=\mathbf{k}_{\bar{V}} \in \mathrm{D}^{\mathrm{b}}\left(\mathbf{k}_{M \times U \times]-\varepsilon, \varepsilon[}\right)$ satisfies the following:

(a) $\quad \operatorname{SS}(\widetilde{L}) \subset(\widetilde{\Lambda} \times I]-\varepsilon, \varepsilon[) \cup T_{M \times U \times]-\varepsilon, \varepsilon[}^{*}(M \times U \times]-\varepsilon, \varepsilon[)$,

(b) $\left.\widetilde{L}\right|_{M \times U \times\{0\}} \simeq \mathbf{k}_{Z_{\varepsilon_{1}}}$,

(c) the projection $M \times U \times]-\varepsilon, \varepsilon[\rightarrow U \times]-\varepsilon, \varepsilon[$ is proper on $\operatorname{Supp}(\widetilde{L})$.

Now we set

$$
K=\left.L^{-1} \circ\right|_{I} \widetilde{L} \in \mathrm{D}^{\mathrm{b}}\left(\mathbf{k}_{U \times U \times]-\varepsilon, \varepsilon[}\right) .
$$

Then $K$ satisfies properties (a) and (b) of Proposition 3.2 when replacing $M$ and $I$ with $U$ and $]-\varepsilon, \varepsilon[$. We deduce in particular

$$
\left.\left.K\right|_{((U \times U) \backslash(A \times A)) \times]-\varepsilon, \varepsilon[} \simeq\left(\mathbf{k}_{\left.\Delta_{M} \times\right]-\varepsilon, \varepsilon[}\right)\right|_{((U \times U) \backslash(A \times A)) \times]-\varepsilon, \varepsilon[} .
$$

Applying Lemma $1.11, K$ extends to $\tilde{K} \in \mathrm{D}^{\mathrm{b}}\left(\mathbf{k}_{M \times M \times]-\varepsilon, \varepsilon[}\right)$ with

$$
\left.\left.\tilde{K}\right|_{((M \times M) \backslash(A \times A)) \times]-\varepsilon, \varepsilon[} \simeq\left(\mathbf{k}_{\left.\Delta_{M} \times\right]-\varepsilon, \varepsilon[}\right)\right|_{((M \times M) \backslash(A \times A)) \times]-\varepsilon, \varepsilon[},
$$

and $\tilde{K} \in \mathrm{D}^{\mathrm{b}}\left(\mathbf{k}_{M \times M \times]-\varepsilon, \varepsilon[}\right)$ is a quantization of $\Phi$ on $]-\varepsilon, \varepsilon[$.

(B) Gluing (a). Assume that $K^{t_{0}, t_{1}} \in \mathrm{D}^{\mathrm{lb}}\left(\mathbf{k}_{M \times M \times] t_{0}, t_{1}[}\right)$ is a quantization of the isotopy $\left\{\varphi_{t}\right\}_{t \in] t_{0}, t_{1}[}$ for an open interval $\left.J=\right] t_{0}, t_{1}[\subset I$ containing the origin.

Assume that $J \neq I$. We show that there exist an open interval $J^{\prime} \subset I$ and a quantization of the isotopy $\left\{\varphi_{t}\right\}_{t \in J^{\prime}}$ such that $J \subset J^{\prime}$ and $J^{\prime} \neq J$.

For an interval $\left.I^{\prime} \subset\right] t_{0}, t_{1}\left[\right.$, we write $\left.K^{t_{0}, t_{1}}\right|_{I^{\prime}}$ for $\left.K^{t_{0}, t_{1}}\right|_{M \times M \times I^{\prime}}$.

Assume that $t_{1} \in I$. By applying the result of (A) to the isotopy $\left\{\varphi_{t} \circ \varphi_{t_{1}}^{-1}\right\}_{t \in I}$, there exist $t_{0}<t_{3}<t_{1}<t_{4}$ with $t_{4} \in I$ and a quantization $L^{t_{3}, t_{4}} \in \mathrm{D}^{\mathrm{b}}\left(\mathbf{k}_{M \times M \times] t_{3}, t_{4}}[)\right.$

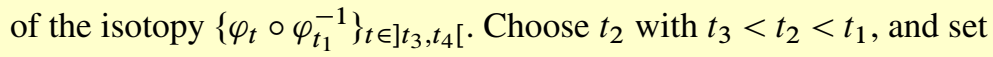

$$
\begin{gathered}
F=\left(\left.K^{t_{0}, t_{1}}\right|_{] t_{3}, t_{1}[}\right) \circ\left(K_{t_{2}}^{t_{0}, t_{1}}\right)^{-1}, \\
F^{\prime}=\left(\left.L^{t_{3}, t_{4}}\right|_{] t_{3}, t_{1}[}\right) \circ\left(L_{t_{2}}^{t_{3}, t_{4}}\right)^{-1} .
\end{gathered}
$$




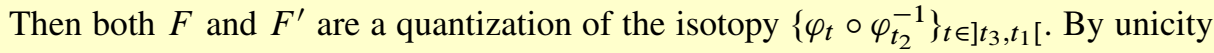
of the quantization (Proposition 3.2), $F$ and $F^{\prime}$ are isomorphic, and hence we have an isomorphism

$$
\left.\left.K^{t_{3}, t_{4}}\right|_{] t_{3}, t_{1}[} \simeq K^{t_{0}, t_{1}}\right|_{] t_{3}, t_{1}[} \quad \text { in } \mathrm{D}^{\mathrm{lb}}\left(\mathbf{k}_{M \times M \times] t_{3}, t_{1}[}\right),
$$

where $K^{t_{3}, t_{4}}=L^{t_{3}, t_{4}} \circ\left(L_{t_{2}}^{t_{3}, t_{4}}\right)^{-1} \circ K_{t_{2}}^{t_{0}, t_{1}} \in \mathrm{D}^{\mathrm{lb}}\left(\mathbf{k}_{M \times M \times] t_{3}, t_{4}[}\right)$. By Lemma 1.11 there exists $K^{t_{0}, t_{4}} \in \mathrm{D}^{\mathrm{lb}}\left(\mathbf{k}_{M \times M \times] t_{0}, t_{4}[}\right)$ such that $\left.K^{t_{0}, t_{4}}\right|_{] t_{0}, t_{1}[} \simeq K^{t_{0}, t_{1}}$ and $\left.K^{t_{0}, t_{4}}\right|_{] t_{3}, t_{4}[} \simeq$

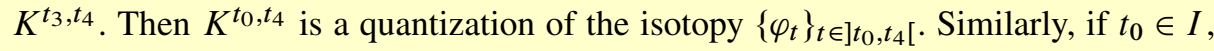
then there exists $t_{-1} \in I$ with $t_{-1}<t_{0}$ and a quantization $K^{t_{-1}, t_{1}}$ on $] t_{-1}, t_{1}[$.

(C) Gluing (b). Consider an increasing sequence of open intervals $I_{n} \subset I$, and assume we have constructed quantizations $K_{n}$ of $\left\{\varphi_{t}\right\}_{t \in I_{n}}$. By unicity in Proposition 3.2 we have $\left.K_{n+1}\right|_{M \times M \times I_{n}} \simeq K_{n}$. Set $J=\bigcup_{n} I_{n}$. By Lemma 1.13 there exists $K_{J} \in \mathrm{D}^{\mathrm{lb}}\left(\mathbf{k}_{M \times M \times J}\right)$ such that $\left.K_{J}\right|_{M \times M \times I_{n}} \simeq K_{n}$. Then $K_{J}$ is a quantization of $\left\{\varphi_{t}\right\}_{t \in J}$.

(D) Consider the set of pairs $\left(J, K_{J}\right)$, where $J$ is an open interval contained in $I$ and containing zero, and $K_{J}$ is a quantization of $\left\{\varphi_{t}\right\}_{t \in J}$. This set, ordered by inclusion, is inductively ordered by $(\mathrm{C})$. Let $\left(J, K_{J}\right)$ be a maximal element. It follows from (B) that $J=I$.

\subsection{Existence of the quantization: General case}

In this section we remove hypothesis (3.3) in Lemma 3.5. We consider $\Phi: \dot{T}^{*} M \times$ $I \rightarrow \dot{T}^{*} M$ which only satisfies (3.1), and we consider $f: \dot{T}^{*} M \times I \rightarrow \mathbb{R}$ and $\Lambda \subset$ $\dot{T}^{*} M \times \dot{T}^{*} M \times T^{*} I$ as above. We define approximations of $\Phi$ by Hamiltonian isotopies satisfying (3.3) such that their quantizations "stabilize" over compact sets, which allows us to define the quantization of $\Phi$.

We consider a $\mathrm{C}^{\infty}$-function $g: M \rightarrow \mathbb{R}$ with compact support. The function

$$
f_{g}: \dot{T}^{*} M \times I \rightarrow \mathbb{R}, \quad(x, \xi, t) \mapsto g(x) f(x, \xi, t)
$$

is homogeneous of degree 1 and has support in $\dot{\pi}_{M}^{-1}(A) \times I$ with $A=\operatorname{supp}(g)$ compact. So its Hamiltonian flow is well defined and satisfies (3.1) and (3.3). We denote it by

$$
\Phi_{g}: \dot{T}^{*} M \times I \rightarrow \dot{T}^{*} M,
$$

and we let $\Lambda_{g} \subset \dot{T}^{*} M \times \dot{T}^{*} M \times T^{*} I$ be the Lagrangian submanifold associated to $\Phi_{g}$ in Lemma A.2. By Lemma 3.5 there exists a unique $K_{g} \in \mathrm{D}^{\mathrm{lb}}\left(\mathbf{k}_{M \times M \times I}\right)$ such that

$$
\operatorname{SS}\left(K_{g}\right) \subset \Lambda_{g} \cup T_{M \times M \times I}^{*}(M \times M \times I) \quad \text { and }\left.\quad K_{g}\right|_{M \times M \times\{0\}} \simeq \mathbf{k}_{\Delta_{M}} .
$$


LEMMA 3.6

Let $U \subset M$ be a relatively compact open subset, and let $J \subset I$ be a relatively compact open subinterval. Then there exists a $\mathrm{C}^{\infty}$-function $g: M \rightarrow \mathbb{R}$ with compact support such that

$$
\left.\Phi_{g}\right|_{\dot{\pi}_{M}(U) \times J}=\left.\Phi\right|_{\dot{\pi}_{M}(U) \times J}
$$

\section{Proof}

We assume without loss of generality that $0 \in J$. Since $\Phi$ is homogeneous, the set $C:=\dot{\pi}_{M}\left(\Phi\left(\dot{\pi}_{M}^{-1}(\bar{U}) \times \bar{J}\right)\right)$ is a compact subset of $M$. We choose a $\mathrm{C}^{\infty}$-function $g: M \rightarrow \mathbb{R}$ with compact support such that $g$ is equal to the constant function 1 on some neighborhood of $C$.

Then for any $p \in \dot{\pi}_{M}^{-1}(U)$ the functions $f$ and $f_{g}$ coincide on a neighborhood of $\gamma_{p, J}:=\left\{\left(\varphi_{t}(p), t\right) ; t \in J\right\} \subset \dot{T}^{*} M \times I$ which is the trajectory of $p$ by the flow $\Phi$. Hence their Hamiltonian vector fields coincide on $\gamma_{p, J}$ as do their flows.

\section{THEOREM 3.7}

We consider $\Phi: \dot{T}^{*} M \times I \rightarrow \dot{T}^{*} M$, and we assume that it satisfies hypothesis (3.1). Then there exists $K \in \mathrm{D}^{\mathrm{lb}}\left(\mathbf{k}_{M \times M \times I}\right)$ satisfying conditions (a) and (b) of Proposition 3.2 .

\section{Proof}

We consider an increasing sequence of relatively compact open subsets $U_{n} \subset M$, $n \in \mathbb{N}$, and an increasing sequence of relatively compact open subintervals $J_{n} \subset I$, $n \in \mathbb{N}$, such that $\bigcup_{n \in \mathbb{N}} U_{n}=M$ and $\bigcup_{n \in \mathbb{N}} J_{n}=I$. By Lemma 3.6 we can choose $\mathrm{C}^{\infty}$-functions $g_{n}: M \rightarrow \mathbb{R}$ with compact supports such that $\Phi_{g_{n}}$ and $\Phi$ coincide on $\dot{\pi}_{M}\left(U_{n}\right) \times J_{n}$. We let $K_{n} \in \mathrm{D}^{\mathrm{lb}}\left(\mathbf{k}_{M \times M \times I}\right)$ be the quantization of $\Phi_{g_{n}}$. In particular

$$
\begin{aligned}
& \mathrm{SS}\left(K_{n}\right) \cap \dot{T}^{*}\left(M \times U_{n} \times J_{n}\right) \subset \Lambda \cap \dot{T}^{*}\left(M \times U_{n} \times J_{n}\right), \\
& \left.K_{n}\right|_{M \times M \times\{0\}} \simeq \mathbf{k}_{\Delta_{M}} .
\end{aligned}
$$

By Lemma 3.3 we have isomorphisms $\left.\left.K_{n+1}\right|_{M \times U_{n} \times J_{n}} \simeq K_{n}\right|_{M \times U_{n} \times J_{n}}$. Then Lemma 1.13 implies that there exists $K \in \mathrm{D}^{\mathrm{lb}}\left(\mathbf{k}_{\boldsymbol{M} \times \boldsymbol{M} \times I}\right)$ with isomorphisms $\left.\left.K\right|_{M \times U_{n} \times J_{n}} \simeq K_{n}\right|_{M \times U_{n} \times J_{n}}$ for any $n \in \mathbb{N}$. Then $K$ satisfies (a) and (b) of Proposition 3.2 by (3.8).

\section{Remark 3.8}

If $\Phi$ also satisfies (3.3) and $J$ is a relatively compact subinterval of $I$, then the restriction $\left.K\right|_{M \times M \times J}$ belongs to $\mathrm{D}^{\mathrm{b}}\left(\mathbf{k}_{\boldsymbol{M} \times M \times J}\right)$ (see Example 3.11). 


\section{Remark 3.9}

Theorem 3.7 extends immediately when replacing the open interval $I$ with a smooth contractible manifold $U$ with a marked point $u_{0}$. Indeed, consider a homogeneous Hamiltonian isotopy $\Phi: \dot{T}^{*} M \times U \rightarrow \dot{T}^{*} M$ with $\varphi_{u_{0}}=\mathrm{id}_{\dot{T}^{*} M}$. We can construct a Lagrangian submanifold $\Lambda$ of $\dot{T}^{*}(M \times M \times U)$ as in Lemma A.2. Set $\delta=\mathrm{id}_{M \times M} \times$ $\delta_{U}$ where $\delta_{U}: U \rightarrow U \times U$ is the diagonal embedding. One easily sees that $\delta_{\pi} \delta_{d}^{-1}(\Lambda)$ is the graph of a homogeneous symplectic diffeomorphism $\widetilde{\Phi}$ of $\dot{T}^{*}(M \times U)$.

Now let $h: U \times I \rightarrow U$ be a retraction to $u_{0}$ such that $h(U \times\{0\})=\left\{u_{0}\right\}$ and $\left.h\right|_{U \times\{1\}}=\operatorname{id}_{U}$. We set $\Phi_{t}=\Phi \circ\left(\operatorname{id}_{\dot{T}^{*} M} \times h_{t}\right)$, and we apply the above procedure to each $\Phi_{t}$. Then $\left\{\widetilde{\Phi}_{t}\right\}_{t \in I}$ is a homogeneous Hamiltonian isotopy of $\dot{T}^{*}(M \times U)$ with $\widetilde{\Phi}_{0}=\mathrm{id}$, and Theorem 3.7 associates with it a kernel $K \in \mathrm{D}^{\mathrm{lb}}\left(\mathbf{k}_{M \times U \times M \times U \times I}\right)$. One checks that $K$ is supported on $M \times M \times \Delta_{U} \times I$ and that $\left.K\right|_{M \times M \times \Delta_{U} \times\{1\}}$ is the desired kernel.

\section{Example 3.10}

Let $M=\mathbb{R}^{n}$, and denote by $(x ; \xi)$ the homogeneous symplectic coordinates on $T^{*} \mathbb{R}^{n}$. Consider the isotopy $\varphi_{t}(x ; \xi)=(x-t(\xi /|\xi|) ; \xi), t \in I=\mathbb{R}$. Then

$$
\begin{aligned}
& \Lambda_{t}=\{(x, y, \xi, \eta) ;|x-y|=|t|, \xi=-\eta=s(x-y), s t<0\} \text { for } t \neq 0, \\
& \Lambda_{0}=\dot{T}_{\Delta}^{*}(M \times M) .
\end{aligned}
$$

The isomorphisms

$$
\begin{aligned}
& \mathrm{R} \mathscr{H o m}\left(\mathbf{k}_{\Delta \times\{t=0\}}, \mathbf{k}_{M \times M \times \mathbb{R}}\right) \simeq \mathbf{k}_{\Delta \times\{t=0\}}[-n-1], \\
& \mathrm{R} \mathscr{H o m}\left(\mathbf{k}_{\{|x-y| \leq-t\}}, \mathbf{k}_{M \times M \times \mathbb{R}}\right) \simeq \mathbf{k}_{\{|x-y|<-t\}}
\end{aligned}
$$

together with the morphism $\mathbf{k}_{\{|x-y| \leq-t\}} \rightarrow \mathbf{k}_{\Delta \times\{t=0\}}$ induce the morphism $\mathbf{k}_{\Delta \times\{t=0\}}[-n-1] \rightarrow \mathbf{k}_{\{|x-y|<-t\}}$. Hence we obtain

$$
\mathbf{k}_{\{|x-y| \leq t\}} \rightarrow \mathbf{k}_{\Delta \times\{t=0\}} \rightarrow \mathbf{k}_{\{|x-y|<-t\}}[n+1] .
$$

Let $\psi$ be the composition. Then there exists a distinguished triangle in $\mathrm{D}^{\mathrm{b}}\left(\mathbf{k}_{M \times M \times I}\right)$ :

$$
\mathbf{k}_{\{|x-y|<-t\}}[n] \rightarrow K \rightarrow \mathbf{k}_{\{|x-y| \leq t\}} \underset{\psi}{\stackrel{+1}{\longrightarrow}}
$$

We can verify that $K$ satisfies the properties (a) and (b) of Proposition 3.2. From this distinguished triangle, we deduce the isomorphisms in $\mathrm{D}^{\mathrm{b}}\left(\mathbf{k}_{M \times M}\right): K_{t} \simeq \mathbf{k}_{\{|x-y| \leq t\}}$ for $t \geq 0$ and $K_{t} \simeq \mathbf{k}_{\{|x-y|<-t\}}[n]$ for $t<0$.

\section{Example 3.11}

We give an example where the quantization $K \in \mathrm{D}^{\mathrm{lb}}\left(\mathbf{k}_{M \times M \times I}\right)$ of a Hamiltonian 
isotopy does not belong to $\mathrm{D}^{\mathrm{b}}\left(\mathbf{k}_{M \times M \times I}\right)$. Let us take the $n$-dimensional unit sphere $M=\mathbb{S}^{n}(n \geq 2)$ endowed with the canonical Riemannian metric. The metric defines an isomorphism $T^{*} M \simeq T M$ and the length function $f: \dot{T}^{*} M \rightarrow \mathbb{R}$. Then $f$ is a positive-valued function on $\dot{T}^{*} M$ homogeneous of degree 1 . Set $I=\mathbb{R}$, and let $\Phi=\left\{\varphi_{t}\right\}_{t \in I}$ be the Hamiltonian isotopy associated with $f$. Then for $p \in \dot{T}^{*} M$, $\left\{\pi_{M}\left(\varphi_{t}(p)\right)\right\}_{t \in I}$ is a geodesic. For $x, y \in M$, dist $(x, y)$ denotes the distance between $x$ and $y$. Let $a: M \rightarrow M$ be the antipodal map. Then we have $\operatorname{dist}(x, y)+\operatorname{dist}(x$, $\left.y^{a}\right)=\pi$. For any integer $\ell$ we set

$$
C_{\ell}= \begin{cases}\left\{(x, y, t) \in M \times M \times \mathbb{R} ; t \geq \ell \pi \text { and } \operatorname{dist}\left(x, a^{\ell}(y)\right) \leq t-\ell \pi\right\} & \text { if } \ell \geq 0, \\ \{(x, y, t) \in M \times M \times \mathbb{R} ; t<(\ell+1) \pi, \text { and } & \\ \left.\operatorname{dist}\left(x, a^{\ell+1}(y)\right)<-t+(\ell+1) \pi\right\} & \text { if } \ell<0 .\end{cases}
$$

Let $K$ be the quantization of $\Phi$. Then we have

$$
H^{k}(K) \simeq \begin{cases}\mathbf{k}_{C_{\ell}} & \text { if } k=(n-1) \ell \text { for some } \ell \in \mathbb{Z}_{\geq 0}, \\ \mathbf{k}_{C_{\ell}} & \text { if } k=(n-1) \ell-1 \text { for some } \ell \in \mathbb{Z}_{<0}, \\ 0 & \text { otherwise. }\end{cases}
$$

\subsection{Deformation of the microsupport}

We consider $\Phi: \dot{T}^{*} M \times I \rightarrow \dot{T}^{*} M$ satisfying hypothesis (3.1) as in Theorem 3.7; we denote as usual by $\Lambda$ the Lagrangian submanifold associated to its graph and define $\Lambda_{t}$ as in (3.2). Let $S_{0} \subset \dot{T}^{*} M$ be a closed conic subset. We set $S=\Lambda \circ S_{0}$ and $S_{t}=\Lambda_{t} \circ S_{0}$, closed conic subsets of $\dot{T}^{*} M \times T^{*} I$ and $\dot{T}^{*} M$, respectively. We let $i_{t}: M \rightarrow M \times I$ be the natural inclusion for $t \in I$. Consider the functor

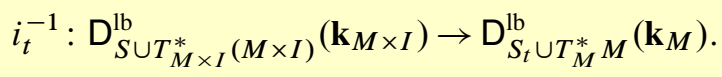

PROPOSITION 3.12

For any $t \in I$ the functor (3.9) is an equivalence of categories. In particular for any $F \in \mathrm{D}^{\mathrm{lb}}\left(\mathbf{k}_{M}\right)$ such that $\mathrm{SS}(F) \subset S_{t} \cup T_{M}^{*} M$ there exists a unique $G \in \mathrm{D}^{\mathrm{lb}}\left(\mathbf{k}_{M \times I}\right)$ such that $i_{t}^{-1}(G) \simeq F$ and $\operatorname{SS}(G) \subset S \cup T_{M \times I}^{*}(M \times I)$. If $\Phi$ also satisfies (3.3) and we replace I by a relatively compact subinterval, then (3.9) induces an equivalence between bounded derived categories.

\section{Proof}

Replacing $\Phi$ by $\Phi \circ \varphi_{t}^{-1}$ we may as well assume that $t=0$. Let $K \in \mathrm{D}^{\mathrm{lb}}\left(\mathbf{k}_{M \times M \times I}\right)$ be the quantization of $\Phi$. Then we obtain the commutative diagram: 


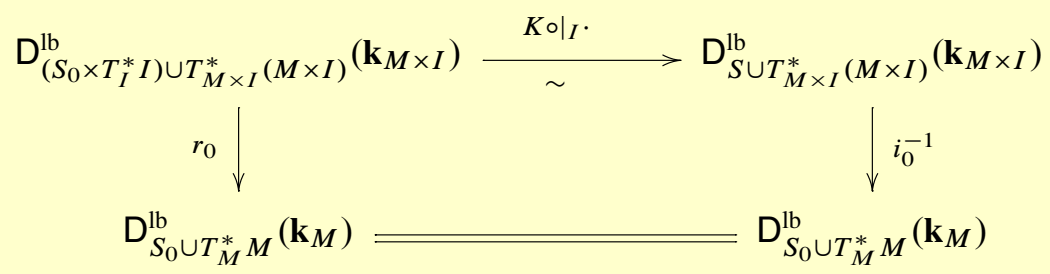

where $r_{0}$ is induced by $i_{0}^{-1}$. It is known that $r_{0}$ is an equivalence of categories, with inverse given by $q_{1}^{-1}$, where $q_{1}: M \times I \rightarrow M$ is the projection. Hence so is the morphism $i_{0}^{-1}$ defined by (3.9).

The last assertion follows from Remark 3.8.

Now we consider a closed conic Lagrangian submanifold $S_{0} \subset \dot{T}^{*} M$ and a deformation of $S_{0}$ indexed by $I, \Psi: S_{0} \times I \rightarrow \dot{T}^{*} M$ as in Definition A.3. We let $S \subset$ $\dot{T}^{*} M \times T^{*} I$ be the corresponding Lagrangian submanifold defined in Lemma A.4. Then Propositions A.5 and 3.12 imply the following result.

\section{COROLLARY 3.13}

We consider a deformation $\Psi: S_{0} \times I \rightarrow \dot{T}^{*} M$ as in Definition A.3, and we assume that it satisfies (A.7). Then for any $t \in I$ the functor (3.9) is an equivalence of categories. Moreover if we replace I by a relatively compact subinterval, it induces an equivalence between the bounded derived categories.

\section{Applications to nondisplaceability}

We denote by $\Phi=\left\{\varphi_{t}\right\}_{t \in I}: \dot{T}^{*} M \times I \rightarrow \dot{T}^{*} M$ a homogeneous Hamiltonian isotopy as in Theorem 3.7. Hence, $\Phi$ satisfies hypothesis (3.1).

Let $F_{0} \in \mathrm{D}^{\mathrm{b}}\left(\mathbf{k}_{M}\right)$. We assume that

$$
F_{0} \text { has a compact support. }
$$

We let $\Lambda \subset \dot{T}^{*}(M \times M \times I)$ be the conic Lagrangian submanifold associated to $\Phi$ in Lemma A.2, and we let $K \in \mathrm{D}^{\mathrm{lb}}\left(\mathbf{k}_{M \times M \times I}\right)$ be the quantization of $\Phi$ on $I$ constructed in Theorem 3.7. We set

$$
\begin{aligned}
& F=K \circ F_{0} \in \mathrm{D}^{\mathrm{lb}}\left(\mathbf{k}_{M \times I}\right), \\
& F_{t_{0}}=\left.F\right|_{\left\{t=t_{0}\right\}} \simeq K_{t_{0}} \circ F_{0} \in \mathrm{D}^{\mathrm{b}}\left(\mathbf{k}_{M}\right) \text { for } t_{0} \in I .
\end{aligned}
$$

Then

$$
\left\{\begin{array}{l}
\operatorname{SS}(F) \subset\left(\Lambda \circ \operatorname{SS}\left(F_{0}\right)\right) \cup T_{M \times I}^{*}(M \times I), \\
\operatorname{SS}(F) \cap T_{M}^{*} M \times T^{*} I \subset T_{M \times I}^{*}(M \times I), \\
\text { the projection } \operatorname{Supp}(F) \rightarrow I \text { is proper. }
\end{array}\right.
$$


The first assertion follows from (1.12), and the second assertion follows from the first. The third one follows from Proposition 3.2(i) and (4.1). In particular we have

$$
\left\{\begin{array}{l}
F_{t} \text { has a compact support in } M, \\
\operatorname{SS}\left(F_{t}\right) \cap \dot{T}^{*} M=\varphi_{t}\left(\operatorname{SS}\left(F_{0}\right) \cap \dot{T}^{*} M\right),
\end{array}\right.
$$

where the last equality follows from (4.3) applied to $\Phi$ and $\left\{\varphi_{t}^{-1}\right\}_{t \in I}$.

\subsection{Nondisplaceability: Homogeneous case}

We consider a $\mathrm{C}^{1}$-map $\psi: M \rightarrow \mathbb{R}$, and we assume that

$$
\text { the differential } d \psi(x) \text { never vanishes. }
$$

Hence the section of $T^{*} M$ defined by $d \psi$,

$$
\Lambda_{\psi}:=\{(x ; d \psi(x)) ; x \in M\},
$$

is contained in $\dot{T}^{*} M$.

\section{THEOREM 4.1}

We consider $\Phi=\left\{\varphi_{t}\right\}_{t \in I}$ satisfying (3.1), $\psi: M \rightarrow \mathbb{R}$ satisfying (4.5), and $F_{0} \in$ $\mathrm{D}^{\mathrm{b}}\left(\mathbf{k}_{M}\right)$ with compact support. We assume that $\mathrm{R} \Gamma\left(M ; F_{0}\right) \neq 0$. Then for any $t \in I$, $\varphi_{t}\left(\operatorname{SS}\left(F_{0}\right) \cap \dot{T}^{*} M\right) \cap \Lambda_{\psi} \neq \emptyset$.

\section{Proof}

Let $F, F_{t}$ be as in (4.2). Then $F_{t}$ has compact support, and $\mathrm{R} \Gamma\left(M ; F_{t}\right) \neq 0$ by Corollary 1.7. Since $\operatorname{SS}\left(F_{t}\right) \subset \varphi_{t}\left(\operatorname{SS}\left(F_{0}\right) \cap \dot{T}^{*} M\right) \cup T_{M}^{*} M$, the result follows from Corollary 1.9.

\section{COROLLARY 4.2}

Let $\Phi=\left\{\varphi_{t}\right\}_{t \in I}$, and let $\psi: M \rightarrow \mathbb{R}$ be as in Theorem 4.1. Let $N$ be a nonempty compact submanifold of $M$. Then for any $t \in I, \varphi_{t}\left(\dot{T}_{N}^{*} M\right) \cap \Lambda_{\psi} \neq \emptyset$.

\subsection{Nondisplaceability: Morse inequalities}

In this subsection and in Section 4.4 below we assume that $\mathbf{k}$ is a field. Let $F_{0} \in$ $\mathrm{D}^{\mathrm{b}}\left(\mathbf{k}_{M}\right)$, and set

$$
S_{0}=\operatorname{SS}\left(F_{0}\right) \cap \dot{T}^{*} M
$$

Now we consider the hypotheses

$\psi$ is of class $\mathrm{C}^{2}$ and the differential $d \psi(x)$ never vanishes, 
$\left\{\begin{array}{l}\text { there exists an open subset } S_{0, \text { reg }} \text { of } S_{0} \text { such that } S_{0 \text {,reg }} \text { is a Lagrangian } \\ \text { submanifold of class } C^{1} \text { and } F_{0} \text { is a simple sheaf along } S_{0, \text { reg }} .\end{array}\right.$

\section{LEMMA 4.3}

Let $\Lambda$ be a smooth Lagrangian submanifold defined in a neighborhood of $p \in \dot{T}^{*} M$, let $G \in \mathrm{D}^{\mathrm{b}}\left(\mathbf{k}_{M}\right)$, and assume that $G$ is simple along $\Lambda$ at $p$. Assume (4.7), and assume that $\Lambda$ and $\Lambda_{\psi}$ intersect transversally at $p$. Set $x_{0}=\pi(p)$. Then

$$
\sum_{j} \operatorname{dim} H^{j}\left(\mathrm{R} \Gamma_{\left\{\psi(x) \geq \psi\left(x_{0}\right)\right\}}(G)\right)_{x_{0}}=1 .
$$

\section{Proof}

By the definition [13, Definition 7.5.4], $\mathrm{R} \Gamma_{\left\{\psi(x) \geq \psi\left(x_{0}\right)\right\}}(G)_{x_{0}}$ is concentrated in a single degree, and its cohomology in this degree has rank one.

In the sequel, for a finite set $A$, we denote by \# $A$ its cardinal.

\section{THEOREM 4.4}

We consider $\Phi=\left\{\varphi_{t}\right\}_{t \in I}$ satisfying (3.1), $\psi: M \rightarrow \mathbb{R}$ satisfying (4.7), and $F_{0} \in$ $\mathrm{D}^{\mathrm{b}}\left(\mathbf{k}_{M}\right)$ with compact support. We also assume (4.8). Let $t_{0} \in I$. Assume that $\Lambda_{\psi} \cap$ $\varphi_{t_{0}}\left(S_{0}\right)$ is contained in $\Lambda_{\psi} \cap \varphi_{t_{0}}\left(S_{0, \mathrm{reg}}\right)$ and the intersection is finite and transversal. Then

$$
\#\left(\varphi_{t_{0}}\left(S_{0}\right) \cap \Lambda_{\psi}\right) \geq \sum_{j} b_{j}\left(F_{0}\right)
$$

\section{Proof}

It follows from Corollary 1.7 that $b_{j}\left(F_{t}\right)=b_{j}\left(F_{0}\right)$ for all $j \in \mathbb{Z}$ and all $t \in I$.

Let $\left\{q_{1}, \ldots, q_{L}\right\}=\Lambda_{\psi} \cap \varphi_{t_{0}}\left(\Lambda_{0}\right), y_{i}=\pi\left(q_{i}\right)$, and set

$$
W_{i}:=\mathrm{R} \Gamma_{\left\{\psi(x) \geq \psi\left(y_{i}\right)\right\}}\left(F_{t_{0}}\right)_{y_{i}} .
$$

By Lemma 4.3, $W_{i}$ is a bounded complex with finite-dimensional cohomologies, and it follows from the Morse inequalities (1.9) that

$$
\sum_{j} b_{j}\left(F_{t_{0}}\right) \leq \sum_{j} \sum_{i} b_{j}\left(W_{i}\right) .
$$

Moreover

$$
\sum_{j} \operatorname{dim} H^{j}\left(\mathrm{R} \Gamma_{\left\{\psi(x) \geq \psi\left(y_{i}\right)\right\}}\left(F_{t_{0}}\right)_{y_{i}}\right)=1 \quad \text { for any } i
$$


and it implies

$$
\sum_{j} \sum_{i} b_{j}\left(W_{i}\right)=\#\left(\operatorname{SS}\left(F_{t_{0}}\right) \cap \Lambda_{\psi}\right)=\#\left(\varphi_{t_{0}}\left(\operatorname{SS}\left(F_{0}\right) \cap \dot{T}^{*} M\right) \cap \Lambda_{\psi}\right) .
$$

COROLLARY 4.5

Let $\Phi=\left\{\varphi_{t}\right\}_{t \in I}$, let $\psi: M \rightarrow \mathbb{R}$ satisfying (4.7), and let $N$ be a compact submanifold of $M$. Let $t_{0} \in I$. Assume that $\varphi_{t_{0}}\left(\dot{T}_{N}^{*} M\right)$ and $\Lambda_{\psi}$ intersect transversally. Then

$$
\#\left(\varphi_{t_{0}}\left(\dot{T}_{N}^{*} M\right) \cap \Lambda_{\psi}\right) \geq \sum_{j} \operatorname{dim} H^{j}\left(N ; \mathbf{k}_{N}\right) .
$$

Proof

Apply Theorem 4.4 with $F_{0}=\mathbf{k}_{N}$.

\section{Remark 4.6}

Corollaries 4.2 and 4.5 extend to the case where $N$ is replaced with a compact submanifold with boundary or even with corners. In this case, one has to replace the conormal bundle $T_{N}^{*} M$ with the microsupport of the constant sheaf $\mathbf{k}_{N}$ on $M$. Note that this microsupport is easily calculated. For Morse inequalities on manifolds with boundaries, see the recent paper [16] and see also [15] and [21] for related results.

\subsection{Nondisplaceability: Nonnegative Hamiltonian isotopies}

Consider as above a manifold $M$, and consider $\Phi: \dot{T}^{*} M \times I \rightarrow \dot{T}^{*} M$ a homogeneous Hamiltonian isotopy; that is, $\Phi$ satisfies (3.1). We define $f: \dot{T}^{*} M \times I \rightarrow \mathbb{R}$ homogeneous of degree 1 and $\Lambda \subset \dot{T}^{*} M \times \dot{T}^{*} M \times T^{*} I$ as in Lemma A.2. The following definition is due to [7] and is used in [4] and [5], where the authors prove Corollary 4.14 below in the particular case where $X$ and $Y$ are points and other related results.

\section{Definition 4.7}

The isotopy $\Phi$ is said to be nonnegative if $\left\langle\alpha_{M}, H_{f}\right\rangle \geq 0$.

Let $\mathrm{eu}_{M}$ be the Euler vector field on $T^{*} M$. Then $\left\langle\alpha_{M}, H_{f}\right\rangle=\mathrm{eu}_{M}(f)$ and since $f$ is of degree 1 we have $\mathrm{eu}_{M}(f)=f$. Hence $\Phi$ is nonnegative if and only if $f$ is a nonnegative valued function. We let $(t, \tau)$ be the coordinates on $T^{*} I$. Then by (A.4) this condition is also equivalent to

$$
\Lambda \subset\{\tau \leq 0\} .
$$

To prove Theorem 4.13, we give several results in sheaf theory. 
PROPOSITION 4.8

Let $N$ be a manifold, and let $I$ be an open interval of $\mathbb{R}$ containing zero. Let $F \in$ $\mathrm{D}^{\mathrm{b}}\left(\mathbf{k}_{N \times I}\right)$, and, for $t \in I$, set $F_{t}=\left.F\right|_{N \times\{t\}} \in \mathrm{D}^{\mathrm{b}}\left(\mathbf{k}_{N}\right)$. Assume that

(a) $\operatorname{SS}(F) \subset\{\tau \leq 0\}$,

(b) $\quad \mathrm{SS}(F) \cap\left(T_{N}^{*} N \times T^{*} I\right) \subset T_{N \times I}^{*}(N \times I)$,

(c) $\operatorname{Supp}(F) \rightarrow I$ is proper.

Then we have the following:

(i) for all $a \leq b$ in I there are natural morphisms $r_{b, a}: F_{a} \rightarrow F_{b}$,

(ii) $\quad r_{b, a}$ induces a commutative diagram of isomorphisms

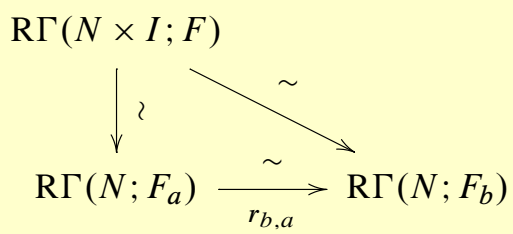

\section{Proof}

By an argument similar to Corollary 1.7 , (b) and (c) imply that $\mathrm{R} \Gamma(N \times I ; F) \rightarrow$ $\mathrm{R} \Gamma\left(N ; F_{t}\right)$ is an isomorphism for any $t \in I$.

For $b \in I$ set $I_{b}=\{t \in I ; t \leq b\}$ and $F^{\prime}=F \otimes \mathbf{k}_{N \times I_{b}}$. Then $F^{\prime}$ also satisfies (a). Hence [13, Proposition 5.2.3] implies that $F^{\prime} \simeq F^{\prime} \circ \mathbf{k}_{D}$, where

$$
\mathrm{D}=\{(s, t) \in I \times I ; t \leq s\} .
$$

We deduce the isomorphisms, for any $a \in I_{b}$ :

$$
F_{a} \simeq F^{\prime} \circ \mathbf{k}_{\{a\}} \simeq F^{\prime} \circ \mathbf{k}_{\mathrm{D}} \circ \mathbf{k}_{\{a\}} \simeq F^{\prime} \circ \mathbf{k}_{[a, b]} \simeq F \circ \mathbf{k}_{[a, b]}
$$

The morphism $r_{b, a}$ is then induced by the morphism $\mathbf{k}_{[a, b]} \rightarrow \mathbf{k}_{\{b\}}$. Hence we obtain a commutative diagram

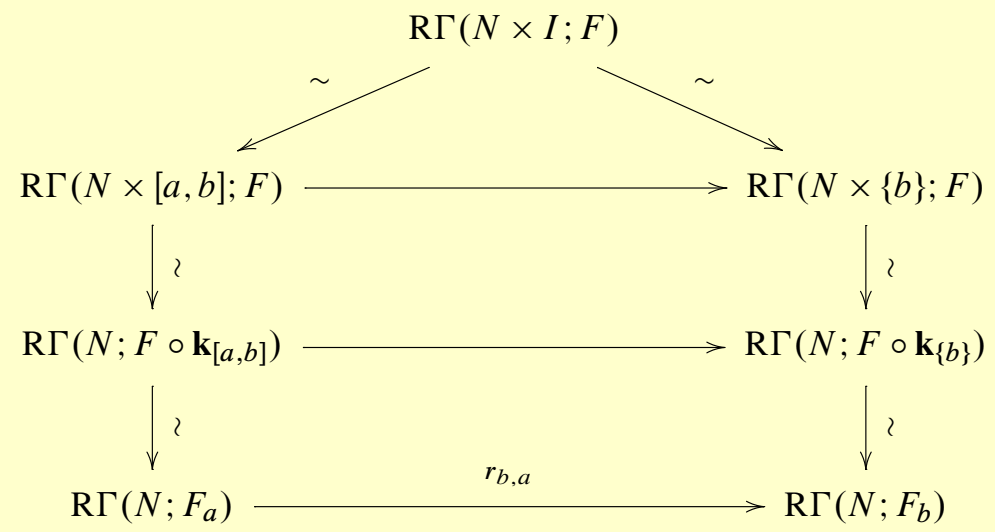


We recall that $\omega_{X}$ denotes the dualizing complex of a manifold $X$.

LEMMA 4.9

Let $M$ be a manifold, and let $X$ be a locally closed subset of $M$. Let $i_{X}: X \rightarrow M$ be the embedding. We assume that the base ring $\mathbf{k}$ is not reduced to $\{0\}$.

(i) Let $F \in \mathrm{D}\left(\mathbf{k}_{M}\right)$, and assume that there exists a morphism $u: F \rightarrow \mathrm{R} i_{X *} \mathbf{k}_{X}$ which induces an isomorphism $H^{0}(M ; F) \stackrel{\sim}{\longrightarrow} H^{0}\left(M ; \mathrm{R} i_{X *} \mathbf{k}_{X}\right)$. Then $X \subset$ $\operatorname{Supp}(F)$.

(ii) Let $G \in \mathrm{D}\left(\mathbf{k}_{M}\right)$, and assume that there exists a morphism $v: i_{X !} \omega_{X} \rightarrow G$ which induces an isomorphism $H_{c}^{0}\left(M ; i_{X !} \omega_{X}\right) \stackrel{\sim}{\longrightarrow} H_{c}^{0}(M ; G)$. Then $X \subset$ $\operatorname{Supp}(G)$.

Proof

(i) Let $x \in X$, and let $i_{x}:\{x\} \hookrightarrow M$ be the inclusion. For $x \in X$, the composition $\mathbf{k} \rightarrow H^{0}\left(M ; \mathrm{R} i_{X *} \mathbf{k}_{X}\right) \rightarrow \mathbf{k}$ is the identity. Hence, in the commutative diagram

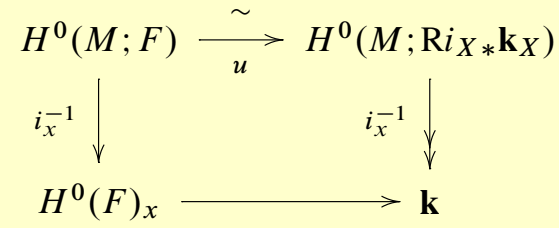

the map $H^{0}(F)_{x} \rightarrow \mathbf{k}$ is surjective. We conclude that $x \in \operatorname{Supp}(F)$.

(ii) For $x \in X$, the composition $H_{\{x\}}^{0}\left(M ; i_{X !} \omega_{X}\right) \rightarrow H_{c}^{0}\left(M ; i_{X !} \omega_{X}\right) \rightarrow \mathbf{k}$ is an isomorphism. Hence in the commutative diagram induced by $v$,

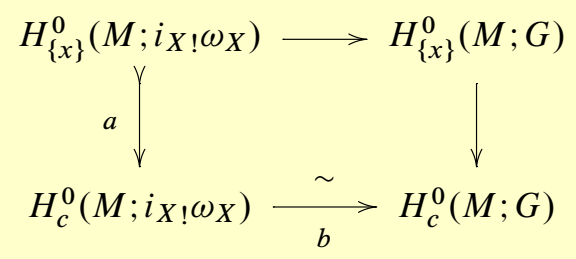

the morphism $a$ is injective and $b$ is bijective. Hence $\mathbf{k} \simeq H_{\{x\}}^{0}\left(M ; i_{X !} \omega_{X}\right) \rightarrow$ $H_{\{x\}}^{0}(M ; G)$ is injective. Therefore $H_{\{x\}}^{0}(M ; G)$ does not vanish and $x \in$ $\operatorname{Supp}(G)$.

LEMMA 4.10

Let $M$ be a noncompact connected manifold, and let $X$ be a compact connected submanifold of $M$. Then we have

(i) the open subset $M \backslash X$ has at most two connected components; 
(ii) assume that there exists a relatively compact connected component $U$ of $M \backslash$ $X$. Then such a connected component is unique, $X$ is a hypersurface, and $X$ coincides with the boundary of $U$.

Proof

(i) We have an exact sequence

$$
H^{0}(M ; \mathbb{C}) \rightarrow H^{0}(M \backslash X ; \mathbb{C}) \rightarrow H_{X}^{1}(M ; \mathbb{C}) .
$$

The last term $H_{X}^{1}(M ; \mathbb{C})$ is isomorphic to $H^{0}\left(X ; H_{X}^{1}\left(\mathbb{C}_{M}\right)\right)$. Since $H_{X}^{1}\left(\mathbb{C}_{M}\right)$ is locally isomorphic to $\mathbb{C}_{X}$ or zero, we have $\operatorname{dim} H_{X}^{1}(M ; \mathbb{C}) \leq 1$. Hence we obtain $\operatorname{dim} H^{0}(M \backslash X ; \mathbb{C}) \leq 2$. Hence $M \backslash X$ has at most two connected components.

(ii) Assume that there exists a relatively compact connected component $U$ of $M \backslash X$. If $M \backslash X$ has another relatively compact connected component $V$, then $M=X \cup U \cup V$ by (i) and it is compact. It is a contradiction. Hence a relatively compact connected component $U$ of $M \backslash X$ is unique if it exists. If $X$ is not a hypersurface, then $M \backslash X$ is connected and not relatively compact. It is a contradiction. Hence $X$ is a hypersurface. Then it is obvious that $X$ coincides with the boundary of $U$.

Until the end of this subsection, we assume that $\Phi=\left\{\varphi_{t}\right\}_{t \in I}: \dot{T}^{*} M \times I \rightarrow \dot{T}^{*} M$ is a nonnegative homogeneous Hamiltonian isotopy.

We define $g: \dot{T}^{*} M \times I \rightarrow \mathbb{R}$ by

$$
g(p, t)=f\left(\varphi_{t}(p)^{a}, t\right) \quad\left(p \in \dot{T}^{*} M, t \in I\right) .
$$

Here $a: \dot{T}^{*} M \rightarrow \dot{T}^{*} M$ is the antipodal map.

\section{LEMMA 4.11}

Let $\Psi$ be the symplectic isotopy given by $\Psi=\left\{a \circ \varphi_{t}^{-1} \circ a\right\}_{t \in I}$. Then we have $\frac{\partial \Psi}{\partial t}=$ $H_{g_{t}}$, and $\Psi$ is a nonnegative Hamiltonian isotopy.

\section{Proof}

Set $\psi_{t}=a \circ \varphi_{t}^{-1} \circ a$. Let $\Lambda$ be the Lagrangian manifold associated to $\Phi$ as in Lemma A.1:

$$
\Lambda=\left\{\left(\varphi_{t}(v), v^{a}, t,-f\left(\varphi_{t}(v), t\right)\right) ; v \in \dot{T}^{*} M, t \in I\right\} .
$$

Then we have

$$
\begin{aligned}
\Lambda & =\left\{\left(w, \varphi_{t}^{-1}(w)^{a}, t,-f(w, t)\right) ; w \in \dot{T}^{*} M, t \in I\right\} \\
& =\left\{\left(w^{a}, \psi_{t}(w), t,-f\left(w^{a}, t\right)\right) ; w \in \dot{T}^{*} M, t \in I\right\} .
\end{aligned}
$$


Since $f\left(w^{a}, t\right)=g\left(\varphi_{t}^{-1}\left(w^{a}\right)^{a}, t\right)$, the set

$$
\left\{\left(w^{a}, \psi_{t}(w), t,-g\left(\psi_{t}(w), t\right)\right) ; w \in \dot{T}^{*} M, t \in I\right\}
$$

is Lagrangian. Hence Lemma A.1 implies that $\partial \Psi / \partial t=g_{t}$. The nonnegativity of $\Psi$ is obvious since $g$ itself is nonnegative.

\section{LEMMA 4.12}

Let $\Lambda_{1}$ and $\Lambda_{2}$ be conic closed Lagrangian submanifolds of $\dot{T}^{*} M$. If either $\varphi_{t}\left(\Lambda_{1}\right) \subset$ $\Lambda_{2}$ for all $t \in[0,1]$ or $\Lambda_{1} \subset \varphi_{t}\left(\Lambda_{2}\right)$ for all $t \in[0,1]$, then $\left.\varphi_{t}\right|_{\Lambda_{1}}=\operatorname{id}_{\Lambda_{1}}$ for all $t \in[0,1]$.

\section{Proof}

(i) Let us treat the case where $\varphi_{t}\left(\Lambda_{1}\right) \subset \Lambda_{2}$ for all $t \in[0,1]$. We may assume that $\Lambda_{1}$ is connected. Then $\varphi_{t}\left(\Lambda_{1}\right)$ is a connected component of $\Lambda_{2}$, and hence does not depend on $t$. Therefore $\varphi_{t}\left(\Lambda_{1}\right)=\Lambda_{1}$ for all $t \in[0,1]$. The hypothesis implies that $H_{f_{t}}=\partial \Phi / \partial t$ is tangent to $\Lambda_{1}$ for all $t \in[0,1]$. By Lemma A.2, $f_{t}=\left\langle\alpha_{M}, H_{f_{t}}\right\rangle$. Since $\Lambda_{1}$ is conic Lagrangian, the Liouville form $\alpha_{M}$ vanishes on the tangent bundle of $\Lambda_{1}$ and we deduce that $f$ is identically zero on $\Lambda_{1} \times[0,1]$.

Since $f_{t}$ is a nonnegative function on $\dot{T}^{*} M$, all points of $\Lambda_{1}$ are minima of $f$. It follows that $d\left(f_{t}\right)=0$ on $\Lambda_{1}$ for all $t \in[0,1]$. Hence $H_{f_{t}}$ also vanishes on $\Lambda_{1}$, and therefore $\left.\varphi_{t}\right|_{\Lambda_{1}}=\mathrm{id}_{\Lambda_{1}}$ for all $t \in[0,1]$.

(ii) Now assume that $\Lambda_{1} \subset \varphi_{t}\left(\Lambda_{2}\right)$ for all $t \in[0,1]$. Set $\psi_{t}=a \circ \varphi_{t}^{-1} \circ a$. Then $\left\{\psi_{t}\right\}_{t \in I}$ is a nonnegative Hamiltonian isotopy by Lemma 4.11, and $\psi_{t}\left(\Lambda_{1}^{a}\right) \subset$ $\Lambda_{2}^{a}$ holds for any $t \in[0,1]$. Hence step (i) implies that $\left.\psi_{t}\right|_{\Lambda_{1}^{a}}=\operatorname{id}_{\Lambda_{1}^{a}}$.

THEOREM 4.13

Let $M$ be a connected and noncompact manifold, and let $X, Y$ be two compact connected submanifolds of $M$. Let $\Phi=\left\{\varphi_{t}\right\}_{t \in I}: \dot{T}^{*} M \times I \rightarrow \dot{T}^{*} M$ be a nonnegative homogeneous Hamiltonian isotopy. Assume that $[0,1] \subset I$ and $\varphi_{1}\left(\dot{T}_{X}^{*} M\right)=\dot{T}_{Y}^{*} M$. Then $X=Y$ and $\left.\varphi_{t}\right|_{\dot{T}_{X}^{*} M}=\operatorname{id}_{\dot{T}_{X}^{*} M}$ for all $t \in[0,1]$ * $^{*}$

\section{Proof}

By Lemma 4.12 it is enough to prove that $X=Y$ and $\varphi_{t}\left(\dot{T}_{X}^{*} M\right) \subset \dot{T}_{X}^{*} M$ for all $t \in[0,1]$.

\footnotetext{
*In an earlier draft of this paper, we only proved the first part of the conclusion of Theorem 4.13, namely, that $X=Y$. We thank Stephan Nemirovski, who asked us the question whether $\left.\varphi_{t}\right|_{\dot{T}_{X}^{*} M}$ is the identity of $\dot{T}_{X}^{*} M$ for all $t \in[0,1]$.
} 
We distinguish two cases (see Lemma 4.10) treated, respectively, in (ii) and (iii) below.

(a) $\quad M \backslash X$ or $M \backslash Y$ has no relatively compact connected component.

(b) Both $X$ and $Y$ are the boundaries of relatively compact connected open subsets $U$ and $V$ of $M$, respectively.

(i) Let $K \in \mathrm{D}^{\text {lb }}\left(\mathbf{k}_{M \times M \times I}\right)$ be the quantization of $\Phi$ on $I$ given by Theorem 3.7. By Proposition 3.2(ii), the convolution with $K_{1}$ gives an equivalence of categories

$$
\mathrm{D}_{T_{X}^{*} M \cup T_{M}^{*} M}^{\mathrm{lb}}\left(\mathbf{k}_{M}\right) \underset{K_{1} \circ}{\sim} \mathrm{D}_{T_{Y}^{*} M \cup T_{M}^{*} M}^{\mathrm{lb}}\left(\mathbf{k}_{M}\right) .
$$

Moreover $\operatorname{SS}(K) \subset \Lambda \cup T_{M \times M \times I}^{*}(M \times M \times I)$, so that $\operatorname{SS}(K) \subset\{\tau \leq 0\}$. We consider $F_{0} \in \mathrm{D}^{\mathrm{b}}\left(\mathbf{k}_{M}\right)$ with compact support. We set

$$
F=K \circ F_{0}, \quad F_{t_{0}}=F \circ \mathbf{k}_{\left\{t=t_{0}\right\}} \quad\left(t_{0} \in I\right) .
$$

Then $F$ satisfies (4.3), and we have $\mathrm{SS}(F) \subset\{\tau \leq 0\}$. Hence we may apply Proposition 4.8, and we deduce that, for all $a, b \in I$ with $a \leq b$, there are natural morphisms $r_{b, a}: F_{a} \rightarrow F_{b}$ which induce isomorphisms $\mathrm{R} \Gamma\left(M ; F_{a}\right) \stackrel{\sim}{\longrightarrow} \mathrm{R} \Gamma\left(M ; F_{b}\right)$.

(ii) Let us assume hypothesis (a). By Lemma 4.11, replacing $\varphi_{t}$ with $a \circ \varphi_{t}^{-1} \circ a$ and $X$ with $Y$ if necessary, we may assume that any of the connected components of $M \backslash X$ is not relatively compact.

(ii-a) Let us show that $X=Y$. There exists $F_{0} \in \mathrm{D}^{\mathrm{lb}}\left(\mathbf{k}_{M}\right)$ such that $F_{1} \simeq \mathbf{k}_{Y}$. We have $F_{0} \simeq K_{1}^{-1} \circ \mathbf{k}_{Y}$ so that $F_{0}$ has compact support. We have also $\operatorname{SS}\left(F_{t}\right) \cap \dot{T}^{*} M=$ $\varphi_{t}\left(\dot{T}_{X}^{*} M\right)$. Since $\operatorname{SS}\left(F_{0}\right) \subset T_{X}^{*} M \cup T_{M}^{*} M, F_{0}$ is locally constant outside $X$. Since $M \backslash X$ has no compact connected component, we deduce $\operatorname{Supp}\left(F_{0}\right) \subset X$. Hence by Lemma 4.9(i), we have $Y \subset \operatorname{Supp}\left(F_{0}\right) \subset X$.

Since $M \backslash X \subset M \backslash Y$ and $M \backslash X$ has no relatively compact connected component, $M \backslash Y$ has also no relatively compact connected component. Hence by interchanging $X$ and $Y$ with the use of Lemma 4.11, we obtain $X \subset Y$. Thus we obtain $X=Y$.

(ii-b) Let us show $\dot{T}_{X}^{*} M \subset \varphi_{t}\left(\dot{T}_{X}^{*} M\right)$. Assuming that $p \in \dot{T}_{X}^{*} M \backslash \varphi_{t}\left(\dot{T}_{X}^{*} M\right)$, let us derive a contradiction. Take a $C^{1}$-function $g$ such that $p=(x ; d g(x))$ and $\left.g\right|_{X}=0$. Since $\operatorname{Supp}\left(F_{0}\right) \cap\{g<0\}=\emptyset$, we obtain $H_{\{g<0\}}^{0}\left(F_{0}\right)_{x} \simeq 0$. Since $d g(x) \notin$ $\operatorname{SS}\left(F_{t}\right)$, the morphism $H^{0}\left(F_{t}\right)_{x} \rightarrow H_{\{g<0\}}^{0}\left(F_{t}\right)_{x}$ is an isomorphism. Then we have a commutative diagram 


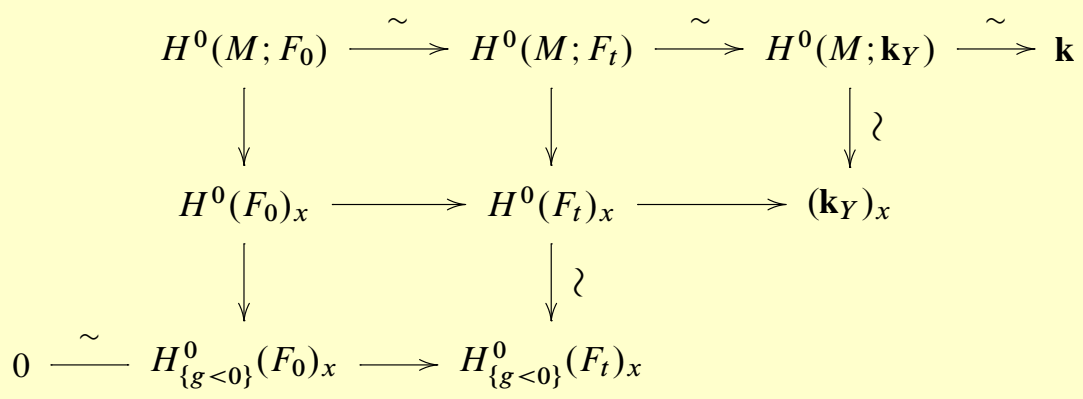

Hence $\mathbf{k} \simeq H^{0}\left(M ; F_{t}\right) \rightarrow H_{\{g<0\}}^{0}\left(F_{t}\right)_{x}$ is a monomorphism and also the zero morphism. This is a contradiction. Thus we obtain the desired result $\dot{T}_{X}^{*} M \subset \varphi_{t}\left(\dot{T}_{X}^{*} M\right)$. Thanks to Lemma 4.12, this completes the proof of the theorem under hypothesis (a).

(iii) Now we assume hypothesis (b). In this case, $X$ and $Y$ are hypersurfaces of $M$. Let $\dot{T}_{Y}^{* \text {,in }} M$ be the "inner" conormal of $Y$, so that $\operatorname{SS}\left(\mathbf{k}_{\bar{V}}\right)=\bar{V} \cup \dot{T}_{Y}^{* \text {,in }} M$ (see Example 1.2).

(iii-a) Let us first prove that $U=V$. As in (ii-a) there exists $F_{0} \in \mathrm{D}^{\mathrm{lb}}\left(\mathbf{k}_{M}\right)$ with compact support such that $F_{1} \simeq \mathbf{k}_{\bar{V}}$. As in (ii-a) we see that $\operatorname{Supp}\left(F_{0}\right) \subset \bar{U}=$ $U \cup X$. Part (i) gives a morphism $r_{1,0}: F_{0} \rightarrow \mathbf{k}_{\bar{V}}$ which induces $H^{0}\left(M ; F_{0}\right) \stackrel{\sim}{\longrightarrow}$ $H^{0}\left(M ; \mathbf{k}_{\bar{V}}\right) \stackrel{\sim}{\longrightarrow} \mathbf{k}$. Hence Lemma 4.9 implies that $\bar{V} \subset \operatorname{Supp}\left(F_{0}\right) \subset \bar{U}$. Then Lemma 4.11 implies the reverse inclusion. Hence $U=V$ and $X=Y$.

(iii-b) Let us prove that $\dot{T}_{X}^{* \text {,in }} M \subset \varphi_{t} \varphi_{1}^{-1}\left(\dot{T}_{X}^{* \text {,in }} M\right)$ for all $t \in[0,1]$. The proof is similar to the one in (ii-b). Assuming that there exist $t \in[0,1]$ and $p \in\left(\dot{T}_{X}^{* \text {,in }} M\right) \backslash$ $\varphi_{t} \varphi_{1}^{-1}\left(\dot{T}_{X}^{*, \text { in }} M\right)$, we derive a contradiction. Write $p=(x ; d g(x))$ for a $\mathrm{C}^{1}$-function $g$ such that $\left.g\right|_{X}=0$. Hence $\{g>0\}$ coincides with $U$ on a neighborhood of $x$. Then we have a commutative diagram

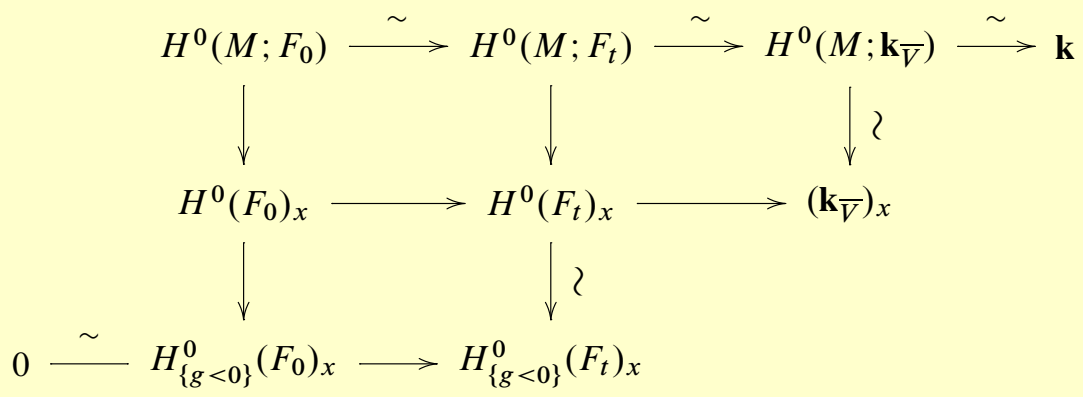

Hence $\mathbf{k} \simeq H^{0}\left(M ; F_{t}\right) \rightarrow H_{\{g<0\}}^{0}\left(F_{t}\right)_{x}$ is a monomorphism and equal to the zero morphism. This is a contradiction. Hence we obtain $\dot{T}_{X}^{* \text {,in }} M \subset \varphi_{t} \varphi_{1}^{-1}\left(\dot{T}_{X}^{* \text {,in }} M\right)$ or, equivalently, $\varphi_{t}^{-1}\left(\dot{T}_{X}^{*, \text { in }} M\right) \subset \varphi_{1}^{-1}\left(\dot{T}_{X}^{* \text {,in }} M\right)$. Hence Lemma 4.12 implies that 
$\left.\varphi_{t}^{-1}\right|_{\dot{T}_{X}^{*, \text { in }} M}=\mathrm{id}_{\dot{T}_{X}^{*, \text { in }} M}$ or $\left.\varphi_{t}\right|_{\dot{T}_{X}^{*, \text { in }} M}=\mathrm{id}_{\dot{T}_{X}^{*, \text { in }} M}$. Lemma 4.11 permits us to apply this to $a \circ \varphi_{t} \circ a$, and we obtain $\left.\varphi_{t}\right|_{a \dot{T}_{X}^{*, \text { in }} M}=\mathrm{id}_{a \dot{T}_{X}^{*, \text { in }} M}$. Thus we obtain $\left.\varphi_{t}\right|_{\dot{T}_{X}^{*} M}=$ $\operatorname{id}_{\dot{T}_{X}^{*} M}$.

\section{COROLLARY 4.14}

Let $M$ be a connected manifold such that the universal covering $\widetilde{M}$ of $M$ is noncompact. Let $X$ and $Y$ be simply connected and compact submanifolds of $M$ with codimension $\geq 2$. Let $\Phi: \dot{T}^{*} M \times I \rightarrow \dot{T}^{*} M$ be a nonnegative homogeneous Hamiltonian isotopy such that $[0,1] \subset I$ and $\varphi_{1}\left(\dot{T}_{X}^{*} M\right)=\dot{T}_{Y}^{*} M$. Then $X=Y$ and $\left.\varphi_{t}\right|_{\dot{T}_{X}^{*} M}=$ $\operatorname{id}_{\dot{T}_{X}^{*} M}$ for all $t \in[0,1]$.

\section{Proof}

Let $q: \widetilde{M} \rightarrow M$ and $p: \dot{T}^{*} \widetilde{M} \rightarrow \dot{T}^{*} M$ be the canonical projections. Let $f: \dot{T}^{*} M \times$ $I \rightarrow \mathbb{R}$ be as in Lemma A.2 for $\Phi$, and set $\tilde{f}:=f \circ\left(p \times \operatorname{id}_{I}\right): \dot{T}^{*} \widetilde{M} \times I \rightarrow \mathbb{R}$. Let $\tilde{\Phi}: \dot{T}^{*} \widetilde{M} \times I \rightarrow \dot{T}^{*} \widetilde{M}$ be the nonnegative homogeneous Hamiltonian isotopy associated with $\tilde{f}$. We set $\tilde{\varphi}_{t}:=\left.\tilde{\Phi}\right|_{\dot{T}^{*} \widetilde{M} \times\{t\}}: \dot{T}^{*} \widetilde{M} \rightarrow \dot{T}^{*} \widetilde{M}$. Then $p \circ \tilde{\varphi}_{t}=\varphi_{t} \circ p$. Let $q^{-1}(X)=\bigsqcup_{j \in J} \tilde{X}_{j}$ and $q^{-1}(Y)=\bigsqcup_{k \in K} \tilde{Y}_{k}$ be the decompositions into connected components. Then by the assumption, $\tilde{X}_{j} \rightarrow X$ and $\tilde{Y}_{k} \rightarrow Y$ are isomorphisms, and hence $\tilde{X}_{j}$ and $\tilde{Y}_{k}$ are connected and compact. Since $p^{-1}\left(\dot{T}_{X}^{*} M\right)=\bigsqcup_{j} \dot{T}_{\tilde{X}_{j}}^{*} \widetilde{M}$, we have

$$
\bigsqcup_{j \in J} \tilde{\varphi}_{1}\left(\dot{T}_{\tilde{X}_{j}}^{*} \widetilde{M}\right)=\bigsqcup_{k \in K} \dot{T}_{\tilde{Y}_{k}}^{*} \widetilde{M}
$$

Since codim $\tilde{X}_{j}, \operatorname{codim} \tilde{Y}_{k}>1$, the unions are decompositions into connected components. So, for a given $j \in J$, there exists $k \in K$ such that $\tilde{\varphi}_{1}\left(\dot{T}_{\tilde{X}_{j}}^{*} \widetilde{M}\right)=\dot{T}_{\tilde{Y}_{k}}^{*} \widetilde{M}$. Hence Theorem 4.13 implies $\tilde{X}_{j}=\tilde{Y}_{k}$ and $\left.\tilde{\varphi}_{t}\right|_{\dot{T}_{\tilde{X}_{j}}^{*}} \widetilde{M}=\mathrm{id}_{\dot{T}_{\tilde{X}_{j}}^{*} \widetilde{M}}$ for all $t \in[0,1]$. Finally we conclude that $X=q\left(\tilde{X}_{j}\right)=q\left(\tilde{Y}_{k}\right)=Y$ and $\left.\varphi_{t}\right|_{\dot{T}_{X}^{*} M}=\operatorname{id}_{\dot{T}_{X}^{*} M}$ for all $t \in[0,1]$.

\subsection{Nondisplaceability: Symplectic case}

In this section we assume that $\mathbf{k}$ is a field. Using Theorems 4.1 and 4.4 we recover a well-known result solving a conjecture by Arnold (see [1], [6], [8], [10], [17]). We first state an easy geometric lemma.

\section{LEMMA 4.15}

Let $p: E \rightarrow X$ be a smooth morphism, let $A, B$ be submanifolds of $X$, and let $A^{\prime}$ be a submanifold of $E$. We assume that $p$ induces a diffeomorphism $\left.p\right|_{A^{\prime}}: A^{\prime} \stackrel{\sim}{\longrightarrow} A$. We set $B^{\prime}=p^{-1}(B)$. Then 
$A^{\prime}$ and $B^{\prime}$ intersect transversally if and only if $A$ and $B$ intersect

transversally.

THEOREM 4.16

Let $N$ be a nonempty compact manifold. Let $\Phi: T^{*} N \times I \rightarrow T^{*} N$ be a Hamiltonian isotopy, and assume that there exists a compact set $C \subset T^{*} N$ such that $\left.\Phi\right|_{\left(T^{*} N \backslash C\right) \times I}$ is the projection on the first factor. We let $c=\sum_{j} \operatorname{dim} H^{j}\left(N ; \mathbf{k}_{N}\right)$, the sum of the Betti numbers of $N$. Then for any $t \in I$ the intersection $\varphi_{t}\left(T_{N}^{*} N\right) \cap T_{N}^{*} N$ is never empty. Moreover its cardinality is at least $c$ whenever the intersection is transversal.

\section{Proof}

(i) We set $M=N \times \mathbb{R}$ and identify $N$ with $N \times\{0\}$. We let $\widetilde{\Phi}: \dot{T}^{*} M \times I \rightarrow$ $\dot{T}^{*} M$ be the homogeneous Hamiltonian isotopy given by Proposition A.6, and we set $\widetilde{\varphi_{t}}=\widetilde{\Phi}(\cdot, t)$.

We apply Theorems 4.1 and 4.4 to $M, \widetilde{\Phi}, F_{0}=\mathbf{k}_{N}$, and $\psi=t$, the projection from $M$ to $\mathbb{R}$. We obtain that the intersection $\widetilde{\varphi}_{t}\left(\dot{T}_{N}^{*} M\right) \cap \Lambda_{\psi}$ is a nonempty set whose cardinality is at least $c$ whenever the intersection is transversal.

(ii) Now we compare $\widetilde{\varphi}_{t}\left(\dot{T}_{N}^{*} M\right) \cap \Lambda_{\psi}$ with the intersection considered in the theorem.

(ii-a) We apply Lemma 4.15 with $X=T^{*} N, E=T^{*} N \times \mathbb{R}^{\times}, p(x, \xi, \sigma)=(x$, $\xi / \sigma), A=T_{N}^{*} N, B=\varphi_{t}\left(T_{N}^{*} N\right)$, and $A^{\prime}=T_{N}^{*} N \times\{1\} \subset E$. We set $\Sigma_{t}:=$ $B^{\prime}=p^{-1}(B)$. We have

$$
\Sigma_{t}=\left\{\left(\sigma \cdot \varphi_{t}(x, 0), \sigma\right) \in T^{*} N \times \mathbb{R}^{\times} ; x \in N, \sigma \in \mathbb{R}^{\times}\right\} .
$$

By Lemma 4.15, $\left(T_{N}^{*} N \times\{1\}\right) \cap \Sigma_{t} \stackrel{\sim}{\longrightarrow} T_{N}^{*} N \cap \varphi_{t}\left(T_{N}^{*} N\right)$, and one of these intersections is transversal if and only if the other one is.

(ii-b) We apply Lemma 4.15 with $X=T^{*} N \times \mathbb{R}^{\times}, E=T^{*} N \times \dot{T}^{*} \mathbb{R}, p(x, \xi$, $s, \sigma)=(x, \xi, \sigma), A=\Sigma_{t}, B=T_{N}^{*} N \times\{1\}$, and $A^{\prime}=\widetilde{\varphi}_{t}\left(\dot{T}_{N}^{*} M\right)$. We must check that the restriction of $p$ to $\widetilde{\varphi}_{t}\left(\dot{T}_{N}^{*} M\right)$ induces an isomorphism $\widetilde{\varphi}_{t}\left(\dot{T}_{N}^{*} M\right) \stackrel{\sim}{\longrightarrow} \Sigma_{t}$. This follows from (4.13) and the identity (see (A.8)):

$$
\widetilde{\varphi}_{t}\left(\dot{T}_{N}^{*} M\right)=\left\{\left(\sigma \cdot \varphi_{t}(x, 0), u(x, 0, t), \sigma\right) ; x \in N, \sigma \in \mathbb{R}^{\times}\right\} .
$$

We see easily that $B^{\prime}=p^{-1}(B)$ is $\Lambda_{\psi}$. Hence Lemma 4.15 implies

$$
\widetilde{\varphi}_{t}\left(\dot{T}_{N}^{*} M\right) \cap \Lambda_{\psi} \stackrel{\sim}{\longrightarrow} \Sigma_{t} \cap\left(T_{N}^{*} N \times\{1\}\right),
$$

and one of these intersections is transversal if and only if the other one is. Together with (ii-a) and (i) this gives the theorem. 


\section{Appendix. Hamiltonian isotopies}

We first recall some notions of symplectic geometry. Let $\mathfrak{X}$ be a symplectic manifold with symplectic form $\omega$. We denote by $\mathfrak{X}^{a}$ the same manifold endowed with the symplectic form $-\omega$. The symplectic structure induces the Hamiltonian isomorphism $\mathbf{h}: T \mathfrak{X} \stackrel{\sim}{\longrightarrow} T^{*} \mathfrak{X}$ by $\mathbf{h}(v)=\iota_{v}(\omega)$, where $\iota_{v}$ denotes the contraction with $v$. To a vector field $v$ on $\mathfrak{X}$ we associate in this way a 1 -form $\mathbf{h}(v)$ on $\mathfrak{X}$. For a $\mathrm{C}^{\infty}$-function $f: \mathfrak{X} \rightarrow \mathbb{R}$ the Hamiltonian vector field of $f$ is by definition $H_{f}:=-\mathbf{h}^{-1}(d f)$.

The vector field $v$ is called symplectic if its flow preserves $\omega$. This is equivalent to $\mathscr{L}_{v}(\omega)=0$, where $\mathscr{L}_{v}$ denotes the Lie derivative of $v$. By Cartan's formula $\left(\mathscr{L}_{v}=d \iota_{v}+\iota_{v} d\right)$ this is again equivalent to $d(\mathbf{h}(v))=0$ (recall that $\left.d \omega=0\right)$. The vector field $v$ is called Hamiltonian if $\mathbf{h}(v)$ is exact, or equivalently $v=H_{f}$ for some function $f$ on $\mathfrak{X}$.

In this section we consider an open interval $I$ of $\mathbb{R}$ containing the origin. We use the following general notation: for a map $u: X \times I \rightarrow Y$ and $t \in I$ we let $u_{t}: X \rightarrow Y$ be the map $x \mapsto u(x, t)$.

\section{A.1. Families of symplectic isomorphisms}

Let $\Phi: \mathfrak{X} \times I \rightarrow \mathfrak{X}$ be a $\mathrm{C}^{\infty}$-map such that $\varphi_{t}:=\Phi(\cdot, t): \mathfrak{X} \rightarrow \mathfrak{X}$ is a symplectic isomorphism for each $t \in I$ and is the identity for $t=0$. The map $\Phi$ induces a timedependent vector field on $\mathfrak{X}$,

$$
v_{\Phi}:=\frac{\partial \Phi}{\partial t}: \mathfrak{X} \times I \rightarrow T \mathfrak{X} .
$$

Since $\varphi_{t}^{*}(\omega)=\omega$ we obtain by derivation $\mathscr{L}_{\left(v_{\Phi}\right)_{t}}(\omega)=0$ for any $t \in I$; that is, $\left(v_{\Phi}\right)_{t}$ is a symplectic vector field. So the corresponding "time-dependent" 1 -form $\beta=\mathbf{h}\left(v_{\Phi}\right): \mathfrak{X} \times I \rightarrow T^{*} \mathfrak{X}$ satisfies $d\left(\beta_{t}\right)=0$ for any $t \in I$. The map $\Phi$ is called a Hamiltonian isotopy if $\left(v_{\Phi}\right)_{t}$ is Hamiltonian, that is, if $\beta_{t}$ is exact for any $t$. In this case, integrating the 1 -form $\beta$ (which is $\mathrm{C}^{\infty}$ with respect to the parameter $t$ ) we obtain a $\mathrm{C}^{\infty}$-function $f: \mathfrak{X} \times I \rightarrow \mathbb{R}$ such that $\beta_{t}=-d\left(f_{t}\right)$. Hence we have

$$
\frac{\partial \Phi}{\partial t}=H_{f_{t}} .
$$

The fact that the isotopy $\Phi$ is Hamiltonian can be interpreted as a geometric property of its graph as follows. For a given $t \in I$ we let $\Lambda_{t}$ be the graph of $\varphi_{t}^{-1}$, and we let $\Lambda^{\prime}$ be the family of $\Lambda_{t}$ 's:

$$
\begin{aligned}
& \Lambda_{t}=\left\{\left(\varphi_{t}(v), v\right) ; v \in \mathfrak{X}^{a}\right\} \subset \mathfrak{X} \times \mathfrak{X}^{a}, \\
& \Lambda^{\prime}=\left\{\left(\varphi_{t}(v), v, t\right) ; v \in \mathfrak{X}^{a}, t \in I\right\} \subset \mathfrak{X} \times \mathfrak{X}^{a} \times I .
\end{aligned}
$$

Then $\Lambda_{t}$ is a Lagrangian submanifold of $\mathfrak{X} \times \mathfrak{X}^{a}$, and we ask whether we can lift $\Lambda^{\prime}$ 
as a Lagrangian submanifold $\Lambda$ of $\mathfrak{X} \times \mathfrak{X}^{a} \times T^{*} I$ so that

$$
\left.\left(\operatorname{id}_{\mathfrak{X} \times \mathfrak{X}^{a}} \times \pi_{I}\right)\right|_{\Lambda}: \Lambda \stackrel{\sim}{\longrightarrow} \Lambda^{\prime} .
$$

LEMMA A. 1

We consider a $\mathrm{C}^{\infty}$-map $\Phi: \mathfrak{X} \times I \rightarrow \mathfrak{X}$ such that $\varphi_{t}$ is a symplectic isomorphism for each $t \in I$, and we use the above notation. Then there exists a Lagrangian submanifold $\Lambda \subset \mathfrak{X} \times \mathfrak{X}^{a} \times T^{*} I$ satisfying (A.3) if and only if $\Phi$ is a Hamiltonian isotopy. In this case the possible $\Lambda$ can be written

$$
\Lambda=\{(\Phi(v, t), v, t,-f(\Phi(v, t), t)) ; v \in \mathfrak{X}, t \in I\},
$$

where the function $f: \mathfrak{X} \times I \rightarrow \mathbb{R}$ is defined by $\left(v_{\Phi}\right)_{t}=H_{f_{t}}$ up to addition of a function depending only on $t$.

We also have, extending notation (1.10) to the case where one manifold is not necessarily a cotangent bundle,

$$
\Lambda_{t}=\Lambda \circ T_{t}^{*} I
$$

\section{Proof}

We write $T^{*} I \simeq I \times \mathbb{R}$. A manifold $\Lambda$ satisfying (A.3) is written

$$
\Lambda=\left\{(\Phi(v, t), v, t, \tau(v, t)) ; v \in \mathfrak{X}^{a}, t \in I\right\}
$$

for some function $\tau: \mathfrak{X}^{a} \times I \rightarrow \mathbb{R}$. Let us write down the condition that $\Lambda$ be Lagrangian. For a given $(v, t) \in \mathfrak{X}^{a} \times I$ and $p=(\Phi(v, t), v, t, \tau(v, t)) \in \Lambda$ the tangent space $T_{p} \Lambda$ is generated by the vectors

$$
\theta_{0}=\left(\left(v_{\Phi}\right)_{t}, 0,1, \frac{\partial \tau}{\partial t}\right) \quad \text { and } \quad \theta_{v}=\left(\left(d \varphi_{t}\right)(v), v, 0,\left(d \tau_{t}\right)(v)\right),
$$

where $v$ runs over $T_{v} \mathfrak{X}^{a}$. Since $\varphi_{t}$ is a symplectic isomorphism the $\theta_{v}$ 's are mutually orthogonal for the symplectic structure of $\mathfrak{X} \times \mathfrak{X}^{a} \times T^{*} I$. Hence $\Lambda$ is Lagrangian if and only if $\theta_{0}$ and $\theta_{v}$ also are orthogonal, which is written

$$
\begin{aligned}
0 & =\omega\left(\left(v_{\Phi}\right)_{t},\left(d \varphi_{t}\right)(v)\right)-\left(d \tau_{t}\right)(v) \\
& =\left(\mathbf{h}\left(\left(v_{\Phi}\right)_{t}\right)-d\left(\tau_{t} \circ \varphi_{t}^{-1}\right)\right)\left(\left(d \varphi_{t}\right)(v)\right) .
\end{aligned}
$$

This holds for all $v \in T_{v} \mathfrak{X}^{a}$ if and only if $\mathbf{h}\left(\left(v_{\Phi}\right)_{t}\right)=d\left(\tau_{t} \circ \varphi_{t}^{-1}\right)$, or equivalently $-H_{\tau_{t} \circ \varphi_{t}^{-1}}=\left(v_{\Phi}\right)_{t}$. 
Exact case. We assume that the symplectic form $\omega$ is exact, and we write $\omega=d \alpha$. We consider $\Phi: \mathfrak{X} \times I \rightarrow \mathfrak{X}$ as above but now we ask that $\varphi_{t}^{*}(\alpha)=\alpha$ for all $t \in I$. Then it is well known (see, e.g., [18, Corollary 9.19]) that $\Phi$ is a Hamiltonian isotopy. More precisely $v_{\Phi}$ is the Hamiltonian vector field of

$$
f=\left\langle\alpha, v_{\Phi}\right\rangle: \mathfrak{X} \times I \rightarrow \mathbb{R}
$$

Indeed the condition on $\varphi_{t}$ implies by derivation $\mathscr{L}_{v_{\Phi}}(\alpha)=0$. Hence Cartan's formula yields

$$
d\left(f_{t}\right)=\mathscr{L}_{\left(v_{\Phi}\right)_{t}}(\alpha)-\iota_{\left(v_{\Phi}\right)_{t}}(\omega)=-\iota_{\left(v_{\Phi}\right)_{t}}(\omega)=-\mathbf{h}\left(\left(v_{\Phi}\right)_{t}\right) .
$$

This holds in particular when $\mathfrak{X}=\dot{T}^{*} M$ for some manifold $M$. We consider $\Phi$ : $\dot{T}^{*} M \times I \rightarrow \dot{T}^{*} M$ such that

$$
\left\{\begin{array}{l}
\varphi_{t} \text { is a homogeneous symplectic isomorphism for each } t \in I, \\
\varphi_{0}=\mathrm{id}_{\dot{T}^{*} M} .
\end{array}\right.
$$

In this case the function $f$ given in (A.5) is homogeneous of degree 1 in the fibers of $\dot{T}^{*} M$ and it is the only homogeneous function such that $\left(v_{\Phi}\right)_{t}=H_{f_{t}}$. So we have the first part of the following lemma.

\section{LEMMA A. 2}

Let $\Phi: \dot{T}^{*} M \times I \rightarrow \dot{T}^{*} M$ satisfying (3.1). Then

(i) $\Phi$ is a Hamiltonian isotopy and there exists a unique conic Lagrangian submanifold $\Lambda$ of $\dot{T}^{*} M \times \dot{T}^{*} M \times T^{*} I$ satisfying (A.3): setting $f=\left\langle\alpha_{M}, \partial \Phi / \partial t\right\rangle$ we have

$$
\Lambda=\left\{(\Phi(x, \xi, t),(x,-\xi),(t,-f(\Phi(x, \xi, t), t))) ;(x, \xi) \in \dot{T}^{*} M, t \in I\right\},
$$

(ii) the set $\Lambda \cup T_{M \times M \times I}^{*}(M \times M \times I)$ is closed in $T^{*}(M \times M \times I)$ and for any $t \in I$ the inclusion $i_{t}: M \times M \rightarrow M \times M \times I$ is noncharacteristic for $\Lambda$ and the graph of $\varphi_{t}$ is $\Lambda_{t}=\Lambda \circ T_{t}^{*} I$.

\section{Proof}

(i) This assertion is already proved.

(ii) In local homogeneous symplectic coordinates $(x, y ; \xi, \eta) \in T^{*}(M \times M)$, $(t ; \tau) \in T^{*} I$, the construction of $\Lambda$ implies that for any compact set $C \subset M \times$ $M \times I$ there exists $D>0$ such that $|\tau| \leq D|\xi|,|\xi| \leq D|\eta|$, and $|\eta| \leq D|\xi|$ for any $(x, y, t ; \xi, \eta, \tau) \in \Lambda \cap \pi_{M \times M \times I}^{-1}(C)$. Hence the same inequalities hold on the closure $\bar{\Lambda}$ of $\Lambda$. Hence if $(x, y, t ; \xi, \eta, \tau) \in \bar{\Lambda} \backslash\left(\dot{T}^{*} M \times \dot{T}^{*} M \times T^{*} I\right)$, then $\xi=\eta=0$ and $\tau=0$, and hence it belongs to the zero-section. 
Hence $\Lambda \cup T_{M \times M \times I}^{*}(M \times M \times I)$ is closed. We also have seen that $\Lambda$ does not meet $T_{M \times M}^{*}(M \times M) \times \dot{T}^{*} I$ which is the noncharacteristicity condition.

\section{A.2. Families of conic Lagrangian submanifolds}

Since the results in this section are well known (they go back to Paulette Libermann), we state them without proofs. Note that we only use them in Corollary 3.13.

\section{Definition A.3}

Let $M$ be a manifold, and let $I$ be an open interval containing zero. Let $S_{0}$ be a closed conic Lagrangian submanifold of $\dot{T}^{*} M$. A deformation of $S_{0}$ indexed by $I$ is the data of a $C^{\infty}$-map $\Psi: S_{0} \times I \rightarrow \dot{T}^{*} M$ such that, setting $\psi_{t}:=\Psi(\cdot, t)$ and $S_{t}=\psi_{t}\left(S_{0}\right)$, we have

(i) $\quad \psi_{0}$ is the identity embedding,

(ii) $\quad \psi_{t}$ is homogeneous for the action of $\mathbb{R}_{>0}$ for each $t \in I$,

(iii) $S_{t}$ is a closed conic Lagrangian submanifold of $\dot{T}^{*} M$ for each $t \in I$,

(iv) the map $S_{0} \times I \rightarrow\left(\dot{T}^{*} M\right) \times I,(s, t) \mapsto(\Psi(s, t), t)$, is an embedding.

We let $S^{\prime}=\left\{(s, t) ; t \in I, s \in S_{t}\right\} \subset\left(\dot{T}^{*} M\right) \times I$ be the image of the embedding in (iv). So it is a closed submanifold of $\left(\dot{T}^{*} M\right) \times I$. Note that $\psi_{t}$ induces a diffeomorphism $\psi_{t}: S_{0} \stackrel{\sim}{\longrightarrow} S_{t}$ for each $t \in I$.

\section{LEMMA A. 4}

Let $S_{0}$ be a closed conic Lagrangian submanifold of $\dot{T}^{*} M$, and let $\Psi: S_{0} \times I \rightarrow$ $\dot{T}^{*} M$ be a deformation of $S_{0}$ as above. Then there exists a unique closed conic Lagrangian submanifold $S \subset \dot{T}^{*}(M \times I)$ such that $\dot{T}^{*}(M \times I) \rightarrow T^{*} M \times I$ induces a diffeomorphism $S \stackrel{\longrightarrow}{\sim} S^{\prime}$.

Moreover for any $t \in I$ the inclusion $i_{t}: M \rightarrow M \times I$ is noncharacteristic for $S$, and we have $S_{t}=S \circ T_{t}^{*} I$.

We remark that $S$, like $S^{\prime}$, only depends on the family of $\left\{S_{t}\right\}_{t}$, not on the parameterization $\Psi$.

For a deformation of a closed conic Lagrangian submanifold we consider a condition similar to (3.3).

There exists a compact subset $A$ of $M$ such that for all $t \in I$ :

$$
\left\{\begin{array}{l}
\left.\psi_{t}\right|_{S_{0} \cap \dot{\pi}_{M}^{-1}(M \backslash A)}=\mathrm{id}_{S_{0} \cap \dot{\pi}_{M}^{-1}(M \backslash A)}, \\
\psi_{t}\left(S_{0} \cap \dot{\pi}_{M}^{-1}(A)\right) \subset \dot{\pi}_{M}^{-1}(A) .
\end{array}\right.
$$




\section{PROPOSITION A.5}

Let $S_{0}$ be a closed conic Lagrangian submanifold of $\dot{T}^{*} M$, and let $\Psi: S_{0} \times I \rightarrow$ $\dot{T}^{*} M$ be a deformation of $S_{0}$ satisfying (A.7).

Then there exists $\Phi: \dot{T}^{*} M \times I \rightarrow \dot{T}^{*} M$ satisfying hypotheses (3.1) and (3.3) such that

$$
\left.\Phi\right|_{S_{0} \times I}=\Psi
$$

\section{A.3. Adding a variable}

In this subsection we recall the link between nonhomogeneous symplectic geometry and homogeneous symplectic geometry with an extra variable.

We denote by $(s, \sigma)$ the coordinates on $T^{*} \mathbb{R}$ with $\sigma d s$ as the Liouville form. For a manifold $M$ we define the map

$$
\rho=\rho_{M}: T^{*} M \times \dot{T}^{*} \mathbb{R} \rightarrow T^{*} M, \quad(x, \xi, s, \sigma) \mapsto(x, \xi / \sigma) .
$$

We consider a Hamiltonian isotopy $\Phi: T^{*} M \times I \rightarrow T^{*} M$ as in Appendix A.1, but we do not assume that it is homogeneous. We show that $\Phi$ lifts to a homogeneous Hamiltonian isotopy of $T^{*} M \times \dot{T}^{*} \mathbb{R}$.

Let $f: T^{*} M \times I \rightarrow \mathbb{R}$ be a function such that $\partial \Phi / \partial t=H_{f_{t}}$ (see (A.2)). We set

$$
\tilde{f}:=(f \circ \rho) \cdot \sigma .
$$

Then $\tilde{f}_{t}$ is a homogeneous function on $T^{*} M \times \dot{T}^{*} \mathbb{R}$ of degree 1 .

PROPOSITION A.6

Let $\Phi: T^{*} M \times I \rightarrow T^{*} M$ be a Hamiltonian isotopy, and let $f$ and $\tilde{f}$ be as above.

(i) Then there exists a homogeneous Hamiltonian isotopy $\widetilde{\Phi}:\left(T^{*} M \times \dot{T}^{*} \mathbb{R}\right) \times$ $I \rightarrow T^{*} M \times \dot{T}^{*} \mathbb{R}$ such that $\partial \widetilde{\Phi} / \partial t=H_{\tilde{f}_{t}}$ and the following diagram commutes:

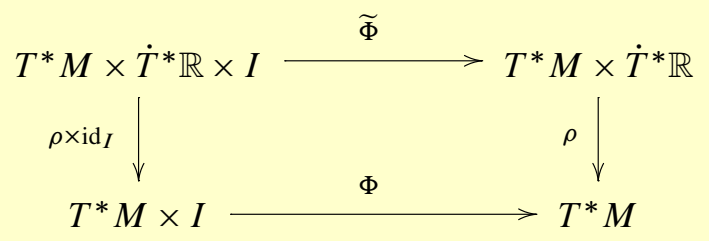

Moreover there exists a $\mathrm{C}^{\infty}$-function $u:\left(T^{*} M\right) \times I \rightarrow \mathbb{R}$ such that

$$
\widetilde{\Phi}(x, \xi, s, \sigma, t)=\left(x^{\prime}, \xi^{\prime}, s+u(x, \xi / \sigma, t), \sigma\right),
$$

where $\left(x^{\prime}, \xi^{\prime} / \sigma\right)=\varphi_{t}(x, \xi / \sigma)$. 
(ii) We assume moreover that $M$ is connected, and $\varphi_{t}$ is the identity outside a compact subset $C \subset T^{*} M$. Then $\widetilde{\Phi}$ extends to a homogeneous Hamiltonian isotopy $\widetilde{\Phi}: \dot{T}^{*}(M \times \mathbb{R}) \times I \rightarrow \dot{T}^{*}(M \times \mathbb{R})$ such that

$$
\widetilde{\Phi}(x, \xi, s, 0, t)=(x, \xi, s+v(t), 0),
$$

for some $\mathrm{C}^{\infty}$-function $v: I \rightarrow \mathbb{R}$.

\section{Proof}

We have to describe the Hamiltonian vector field $H_{\widetilde{f}}$ of $\widetilde{f}$. We denote by $p: T^{*} M \times$ $\dot{T}^{*} \mathbb{R} \rightarrow \dot{T}^{*} \mathbb{R}$ the projection $(x, \xi, s, \sigma) \mapsto(s, \sigma)$. Then $(\rho, p)$ defines an isomorphism

$$
\psi: T^{*} M \times \dot{T}^{*} \mathbb{R} \stackrel{\sim}{\longrightarrow} T^{*} M \times \dot{T}^{*} \mathbb{R}
$$

For a point $q=(x, \xi, s, \sigma) \in T^{*} M \times \dot{T}^{*} \mathbb{R}, \psi$ defines an isomorphism on the tangent spaces:

$$
\begin{aligned}
d \psi & =d \rho_{q} \times d p_{q}: T_{q}\left(T^{*} M \times T^{*} \mathbb{R}\right) \\
& \stackrel{\sim}{\longrightarrow} T_{(x, \xi / \sigma)}\left(T^{*} M\right) \oplus T_{(s, \sigma)}\left(T^{*} \mathbb{R}\right) .
\end{aligned}
$$

Setting $\omega_{M}=d \alpha_{M}$, where $\alpha_{M}$ is the Liouville form on $T^{*} M$, we have

$$
\begin{aligned}
& \left.\alpha_{M \times \mathbb{R}}\right|_{T^{*} M \times \dot{T}^{*} \mathbb{R}}=\sigma \rho^{*}\left(\alpha_{M}\right)+p^{*}\left(\alpha_{\mathbb{R}}\right), \\
& \left.\omega_{M \times \mathbb{R}}\right|_{T^{*} M \times \dot{T}^{*} \mathbb{R}}=\sigma \rho^{*}\left(\omega_{M}\right)+p^{*}\left(\omega_{\mathbb{R}}\right)+d \sigma \wedge \rho^{*}\left(\alpha_{M}\right) .
\end{aligned}
$$

In the sequel we fix $t$, and we set $\tilde{f}_{t}=\widetilde{f}(\cdot, t)$ and $f_{t}=f(\cdot, t)$. Then $H_{\tilde{f}_{t}}$ is determined by $\iota_{\tilde{f}_{t}}\left(\omega_{M \times \mathbb{R}}\right)=-d \tilde{f}_{t}$. We decompose $\left(H_{\tilde{f}_{t}}\right)_{q}=v_{M}+v_{\mathbb{R}}$ according to (A.11), and we also use the decomposition of $T_{q}^{*}\left(T^{*} M \times T^{*} \mathbb{R}\right)$ induced by (A.11). Then we find

$$
\iota_{\tilde{f}_{t}}\left(\omega_{M \times \mathbb{R}}\right)=\left(\sigma \iota_{v_{M}}\left(\omega_{M}\right)+\left\langle v_{\mathbb{R}}, d \sigma\right\rangle \alpha_{M}\right)+\left(\iota_{v_{\mathbb{R}}}\left(\omega_{\mathbb{R}}\right)-\left\langle v_{M}, \alpha_{M}\right\rangle d \sigma\right) .
$$

Since $d \tilde{f_{t}}=\sigma \rho^{*} d f_{t}+\rho^{*}\left(f_{t}\right) d \sigma$ we obtain

$$
\begin{aligned}
-d f_{t} & =\iota_{v_{M}}\left(\omega_{M}\right)+\sigma^{-1}\left\langle v_{\mathbb{R}}, d \sigma\right\rangle \alpha_{M}, \\
-\rho^{*}\left(f_{t}\right) d \sigma & =\iota_{v_{\mathbb{R}}}\left(\omega_{\mathbb{R}}\right)-\left\langle v_{M}, \alpha_{M}\right\rangle d \sigma .
\end{aligned}
$$

The second equality gives $v_{\mathbb{R}}=a \frac{\partial}{\partial s}$ for some function $a$. Then we have $\left\langle v_{\mathbb{R}}, d \sigma\right\rangle=0$, which implies $v_{M}=H_{f_{t}}$ by the first equality, and hence $a=\left(f_{t}-\left\langle H_{f_{t}}, \alpha_{M}\right\rangle\right) \circ \rho=$ $\left(f_{t}-\mathrm{eu}_{M}\left(f_{t}\right)\right) \circ \rho$. Finally, letting $g:=f-\mathrm{eu}_{M}(f)$ be a function on $T^{*} M \times I$, we 
obtain

$$
\psi_{*}\left(H_{\tilde{f}_{t}}\right)=H_{f_{t}}+\rho^{*}\left(g_{t}\right) \frac{\partial}{\partial s} .
$$

Let us define $u: T^{*} M \times I \rightarrow \mathbb{R}$ by the differential equation

$$
\left\{\begin{array}{l}
\frac{\partial u}{\partial t}=g_{t} \circ \varphi_{t} \\
\left.u\right|_{t=0}=0
\end{array}\right.
$$

We define $\widetilde{\Phi}$ by (A.8). Then, we can see easily that

$$
\frac{\partial \widetilde{\Phi}}{\partial t}=H_{\tilde{f}_{t}}
$$

Hence $\widetilde{\Phi}$ is the desired homogeneous Hamiltonian isotopy.

(ii) The functions $f_{t}$ and $g_{t}$ are constant functions outside $C$. Hence $u_{t}$ is also a constant function outside $C$ taking the value $v(t)$. Then $\widetilde{\Phi}$ extends to a homogeneous Hamiltonian isotopy $\widetilde{\Phi}: \dot{T}^{*}(M \times \mathbb{R}) \times I \rightarrow \dot{T}^{*}(M \times \mathbb{R})$ by (A.9).

Acknowledgments. We have been very much stimulated by the interest of Claude Viterbo for the applications of sheaf theory to symplectic topology, and it is a pleasure to thank him here.

We are also extremely grateful to Emmanuel Ferrand who pointed out to us the crucial fact that the Arnold nondisplaceability problem could be treated through homogeneous symplectic methods.

\section{References}

[1] M. CHAPERON, Quelques questions de geométrie symplectique (d'après, entre autres, Poincaré, Arnold, Conley et Zehnder), Astérisque 105-106, 231-249, Séminaire Bourbaki 1982/1983, Soc. Math. France, Montrouge, 1983. MR 0728991 (202, 235)

[2] - "On generating families" in The Floer Memorial Volume, Progr. Math. 133, Birkhäuser, Basel, 1995, 283-296. MR 1362831 (202)

[3] Y. CHEKANOV, Critical points of quasifunctions and generating families of Legendrian manifolds, Funct. Anal. Appl. 30 (1996), 118-128. MR 1402081 (202)

[4] V. CHERNOV and S. NEMIROVSKI, Non-negative Legendrian isotopy in $S T^{*} M$, Geom. Topol. 14 (2010), 611-626. MR 2602847 (203, 228)

[5] V. COLIN, E. FERRAND, and P. PUSHKAR, Positive isotopies of Legendrian submanifolds and applications, preprint, arXiv:1004.5263v1 [math.SG] (203, 228) 
[6] C. CONLEY and E. ZEHNDER, The Birkhof-Lewis fixed point theorem and a conjecture of V. I. Arnol'd, Invent. Math. 73 (1983), 33-49. MR 0707347 (202, 235)

Y. ELIASHBERG, S. S. KIM, and L. POLTEROVICH, Geometry of contact transformations and domains: Orderability versus squeezing, Geom. Topol. 10 (2006), 1635-1747. MR 2284048 (228)

[8] E. FERRAND, "On a theorem of Chekanov" in Symplectic Singularities and Geometry of Gauge Fields, Banach Center Publ. 39, Polish Acad. Sci., Warsaw, 1997, 39-48. MR 1458647 (202, 235)

[9] S. GUILLERMOU and P. SCHAPIRA, Microlocal theory of sheaves and Tamarkin's non displaceability theorem, preprint, arXiv:1106.1576v1 [math.SG] (202)

[10] H. HOFER, Lagrangian embeddings and critical point theory, Ann. Inst. H. Poincaré Anal. Non Linéaire 2 (1985), 407-462. MR 0831040 (202, 235)

[11] M. KASHIWARA and P. SCHAPIRA, Micro-support des faisceaux: Applications aux modules différentiels, C. R. Acad. Sci. Paris Série I Math. 295 (1982), 487-490. MR 0684086 (201)

[12] - Microlocal Study of Sheaves, Astérisque 128, Soc. Math. France, Montrouge, 1985. MR 0794557 (201)

[13] - Sheaves on Manifolds, Grundlehren Math. Wiss. 292, Springer, Berlin, 1990. MR 1074006 (201, 203, 204, 205, 206, 207, 208, 211, 212, 213, 227, 229)

[14] Categories and Sheaves, Grundlehren Math. Wiss. 332, Springer, Berlin, 2006. MR 2182076 ()

[15] R. KASTURIRANGAN and Y.-G. OH, Floer homology of open subsets and a relative version of Arnold's conjecture, Math. Z. 236 (2001), 151-189. MR 1812454 (202, 228)

[16] F. LAUDENBACH, A Morse complex on manifolds with boundary, preprint, arXiv:1003.5077v2 [math.SG] (228)

[17] F. LAUDENBACH and J.-C. SIKORAV, Persistance d'intersection avec la section nulle au cours d'une isotopie hamiltonienne dans un fibré cotangent, Invent. Math. 82 (1985), 349-357. MR 0809719 (202, 235)

[18] D. MCDUFF and D. SALAMON, Introduction to symplectic topology, Oxford Math. Monogr. Oxford Univ. Press, New York, 1995. MR 1373431 (239)

[19] D. NADLER, Microlocal branes are constructible sheaves, preprint, arXiv:math/0612399v2 [math.SG] (202)

[20] D. NADLER and E. ZASLOW, Constructible sheaves and the Fukaya category, J. Amer. Math. Soc. 22 (2009), 233-286. MR 2449059 (202)

[21] Y.-G. OH, "Naturality of Floer homology of open subsets in Lagrangian intersection theory" in The Third Pacific Rim Geometry Conference (Seoul, 1996), Monogr. Geom. Topol. 25, Int. Press, Cambridge, Mass., 1998, 261-280. MR 1751076 (202, 228)

[22] P. POLESELLO and P. SCHAPIRA, Stacks of quantization-deformation modules over complex symplectic manifolds, Int. Math. Res. Notices 2004, no. 49, 2637-2664. MR 2077680 (202)

[23] P. SCHAPIRA and N. TOSE, Morse inequalities for R-constructible sheaves, Adv. Math. 93 (1992), 1-8. MR 1160840 (207) 
[24] D. TAMARKIN, Microlocal conditions for non-displaceability, arXiv:0809.1584v1 [math.SG] (202)

\section{Guillermou}

Institut Fourier, Université de Grenoble I, 38402 Saint Martin d'Hères Cedex, France; Stephane.Guillermou@ujf-grenoble.fr

\section{Kashiwara}

Research Institute for Mathematical Sciences, Kyoto University, Kyoto 606-8502, Japan; masaki@kurims.kyoto-u.ac.jp

\section{Schapira}

Institut de Mathématiques, Université Pierre et Marie Curie, Paris Cedex 05, 75252, France; schapira@math.jussieu.fr 